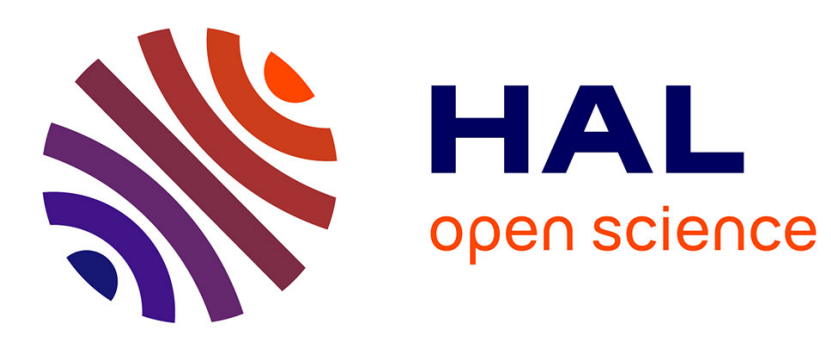

\title{
A slender dielectric body embedded in an arbitrary external potential
}

Antoine Sellier

\section{To cite this version:}

Antoine Sellier. A slender dielectric body embedded in an arbitrary external potential. Journal of Applied Mathematics, 2001, 66 (2), pp.149-173. 10.1093/imamat/66.2.149 . hal-01025343

HAL Id: hal-01025343

https://hal-polytechnique.archives-ouvertes.fr/hal-01025343

Submitted on 11 Sep 2014

HAL is a multi-disciplinary open access archive for the deposit and dissemination of scientific research documents, whether they are published or not. The documents may come from teaching and research institutions in France or abroad, or from public or private research centers.
L'archive ouverte pluridisciplinaire HAL, est destinée au dépôt et à la diffusion de documents scientifiques de niveau recherche, publiés ou non, émanant des établissements d'enseignement et de recherche français ou étrangers, des laboratoires publics ou privés. 


\title{
A slender dielectric body embedded in an arbitrary external potential
}

\author{
A. SELLIER \\ LADHYX, Ecole polytechnique, 91128, Palaiseau Cedex, France
}

[Received 7 June 1999]

\begin{abstract}
This paper determines the electrostatic potential and field taking place both inside and outside a slender dielectric body embedded in a given potential $\phi_{0}$. This task is actually reduced to the determination of the occurring polarization surface-charge density $q$ which depends on $\phi_{0}$, on the body shape but also on the ratio $\delta=\epsilon_{2} / \epsilon_{1}$ of the dielectic constants ( $\epsilon_{2}$ outside and $\epsilon_{1}$ inside the body). The adopted procedure consists in asymptotically expanding and inverting (with respect to the small slenderness ratio of the body) the wellknown Fredholm boundary integral equation of the second kind governing the function $q$. The technical difficulties such an approach encounters are bypassed by employing a systematic formula in getting the asymptotic estimate of certain integrals depending upon a small parameter. Contrary to other works in the field, this method authorizes us to handle the case of non-axisymmetric slender bodies. As an illustration the theory is applied to a body of elliptical cross-section and comparisons are presented for a slender dielectric ellipsoid embedded in a special potential $\phi_{0}$ for which the exact density $q$ is obtained in a closed form.
\end{abstract}

Keywords: asymptotic expansions; integral equations; slender dielectric body.

\section{Introduction}

We consider a slender dielectric body embedded in a specified but arbitrary electrostatic potential field $\phi_{0}$. Since it further provides the electrostatic potential and field both around and inside the body we look for the polarization charge density taking place on the body surface. This unknown density, denoted by $q$, depends on the applied potential $\phi_{0}$, on the body shape (especially on its small slenderness ratio $\epsilon$ ) and also on the positive ratio $\delta$ of the dielectric constants outside and inside the body. For a slender body of revolution Barshinger \& Geer (1987) derived the asymptotic behaviour of $q$ with respect to $\epsilon$ for the three different circumstances $\delta \geqslant O(1), \delta=O(\epsilon)$ and $\delta=O\left(\epsilon^{2}\right)$ in case of an axisymmetric external potential $\phi_{0}$ and for any positive $\delta$ for an asymmetric applied potential. The basic density $q$ was actually deduced from the asymptotic estimates these authors first built for the electrostatic potential by using inside the body an unknown Taylor series $(T s)$ in the radial distance from the axis of the body and outside unknown point singularities $(s)$ placed along a part of this axis inside the body. Those unknown quantities (Ts and $s$ ) are obtained by inverting a pair of coupled integral equations. Representating the potential outside the body by spreading point singularities inside but somewhere on its axis has been pioneered by Handelsman \& Keller $(1967 \mathrm{a}, \mathrm{b})$ and further by several workers (see for instance Moran (1963) and Geer $(1974,1975,1976)$ ) in other contexts for a slender body of revolution or a thin two-dimensional body. At least for the electrostatic 
case, Cade (1994), however, outlined that this procedure sometimes yields an ill-posed integral equation. More precisely, the integral equation may have no solution for some applied potential $\phi_{0}$ (think about the example pertaining to Cade (1994, Fig. 2) even if the body surface (as assumed by Moran (1963) and others) is globally analytic. As far as the author knows, both necessary and sufficient conditions for the derived integral equation to be well-posed have not been clearly stated and proved.

The aim of this paper is to present an alternative method free from the previous potential drawback (that is, a well-posed formulation) and also valid for a 'straight' (see Section 2) slender body of arbitrary cross-section. In the spirit of Sellier $(1997,1999)$ this task is achieved by asymptotically inverting with respect to the slenderness ratio $\epsilon$ a well-posed Fredholm boundary integral equation this time governing the polarization charge density $q$. As will be explained, the asymptotic estimate of the density $q$ is obtained, as $\epsilon$ goes to zero, for three different cases: $\delta-1 \geqslant O(1), \delta=O(1)$ and $0<1-\delta=O(1), 0<|\delta-1|=o(1)$. The case $\delta=0$ of a perfectly conducting and isolated slender body of zero total charge has been handled by Sellier (1999) (this paper also considers the general case of a non-zero total charge). Note that Sellier (1997) dealt with the potential flow of an inviscid fluid about a 'straight' slender body which is not necessarily of revolution but admits pointed ends and lies in a uniform ambient flow. The present work makes it possible to extend these results to the general case of an arbitrary ambient flow and rounded ends by choosing $\delta \rightarrow \infty$.

This paper is organized as follows. The well-posed boundary integral formulation is proposed in Section 2 whilst Section 3 deals with the key asymptotic expansion of the integral equation by means of a systematic formula previously established in Sellier (1996) and briefly reported in 6. One thereafter deduces in Section 4 asymptotic estimates both for the polarization surface-charge density $q$ and related quantities of interest. In order to discuss the validity of the proposed approach, in Section 5 we apply the theory to the case of a slender body of elliptical cross-section. This application indeed authorizes us to recover the conclusions of Barshinger \& Geer (1987) for a dielectric body of revolution and also to compare, for a slender dielectric ellipsoid embedded in a specific electrostatic potential, our results with the asymptotic behaviour of the exact solution one can derive (as detailed in 6) in a closed form.

\section{The boundary integral formulation}

The 'straight' and slender dielectric body of interest $\mathcal{A}^{\prime}$ is an open, simply connected and bounded subset of $\mathbb{R}^{3}$ whose boundary $\partial \mathcal{A}^{\prime}$ is smooth. Accordingly, for each point $M$ of $\partial \mathcal{A}^{\prime}$ one introduces the usual outward normal unit vector $\mathbf{n}(M)$ and the body admits rounded ends $O^{\prime}$ and $E^{\prime}$ (see Fig. 1).

In order to locate each point $M$ of the space we resort to a Cartesian set of coordinates $\left(O^{\prime}, x^{\prime}, y^{\prime}, z^{\prime}\right)$ such that $\mathbf{e}_{z}=\mathbf{O}^{\prime} \mathbf{E}^{\prime} / O^{\prime} E^{\prime}$. If $L=O^{\prime} E^{\prime}$ and $e^{2}=\operatorname{Max}\left(x^{\prime 2}+y^{\prime 2}\right)$ for $M \in \partial \mathcal{A}^{\prime}$ then the slenderness ratio $\epsilon=e / L$ obeys $0<\epsilon \ll 1$. Our slender body is 'straight' in the sense that it collapses to the straight segment $O^{\prime} E^{\prime}$ as $\epsilon$ goes to zero.

Both media $\mathcal{A}^{\prime}$ and $\mathbb{R}^{3} \backslash\left(\mathcal{A}^{\prime} \cup \partial \mathcal{A}^{\prime}\right)$ are linear, homogeneous and isotropic dielectrics with $\epsilon_{1}$ and $\epsilon_{2}$ respectively denoting the associated dielectric constants. The slender dielectric body $\mathcal{A}^{\prime}$ is embedded in an imposed electrostatic potential $\phi_{0}$ induced by fixed charges lying far from the body and belonging to a subset $\Omega$ of $\mathbb{R}^{3} \backslash\left(\mathcal{A}^{\prime} \cup \partial \mathcal{A}^{\prime}\right)$. In such 


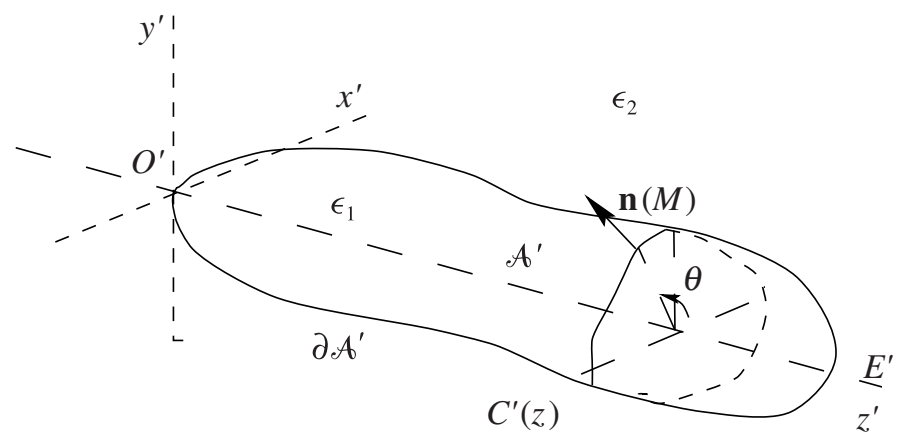

FIG. 1. Sketch of a 'straight' slender dielectric body with an indication of the employed coordinate systems.

circumstances (see, for instance, Jackson (1975)), a polarization surface-charge density $q$ takes place on $\partial \mathcal{A}^{\prime}$ whereas the total volume-charge distribution remains zero outside $\Omega$. Hence, for $M \in \mathbb{R}^{3} \backslash \Omega$, the total electrostatic potential $\phi$ and field $\mathbf{E}$ are written

$$
\phi(M)=\phi_{0}(M)+\iint_{\partial \mathcal{A}^{\prime}} \frac{q(P) \mathrm{d} S_{P}^{\prime}}{4 \pi \epsilon_{0} P M} ; \quad \mathbf{E}(M)=-\operatorname{grad}[\phi](M),
$$

where $\epsilon_{0}$ designates the free space permittivity. Thus, the functions $\phi_{0}$ and $\phi$ are respectively harmonic in $\mathbb{R}^{3} \backslash \Omega$ and $\mathbb{R}^{3} \backslash\left(\Omega \cup \partial \mathcal{A}^{\prime}\right)$ and $\phi \sim \phi_{0}$ far from the body. The representation (2.1) also ensures that $\phi$ is continuous together with its tangential derivatives across the surface $\partial \mathcal{A}^{\prime}$. The density $q$ is determined by imposing the continuity of the displacement field across $\partial \mathcal{A}^{\prime}$ (see Kellogg (1953) and Jackson (1975)), that is

$$
\epsilon_{1} \mathbf{E}_{1}(M) \cdot \mathbf{n}(M)=\epsilon_{2} \mathbf{E}_{2}(M) \cdot \mathbf{n}(M) \quad \text { for } M \in \partial \mathcal{A}^{\prime},
$$

where $\mathbf{E}_{1}(M)$ and $\mathbf{E}_{2}(M)$ respectively denote the limit value of $\mathbf{E}(P)$ as $P$ approaches the point $M$ of $\partial \mathcal{A}^{\prime}$ from the internal or the external side of the body surface. More precisely, our representation (2.1) yields the following (see Kellogg (1953)). If $\mathbf{E}_{0}=-\operatorname{grad}\left[\phi_{0}\right]$ then for $i \in\{1,2\}$,

$$
\left[\mathbf{E}_{i}-\mathbf{E}_{0}\right](M) \cdot \mathbf{n}(M)=(-1) \frac{i q(M)}{2 \epsilon_{0}}+\iint_{\partial \mathcal{A}^{\prime}} \frac{\mathbf{P M} \cdot \mathbf{n}(M) q(P) \mathrm{d} S_{P}^{\prime}}{4 \pi \epsilon_{0} P M^{3}}, \quad M \in \partial \mathcal{A}^{\prime}
$$

Observe that the integral arising on the right-hand side of (2.3) is regular. Since it has already been addressed in Sellier (1999) the special case $\delta:=\epsilon_{2} / \epsilon_{1}=0$ is disregarded. For $\delta>0$, the combination of relations (2.2), (2.3) yields

$$
\frac{q(M)}{\epsilon_{0}}=\left[\mathbf{E}_{2}-\mathbf{E}_{1}\right](M) \cdot \mathbf{n}(M)=\frac{1-\delta}{\delta} \mathbf{E}_{1}(M) \cdot \mathbf{n}(M), \quad M \in \partial \mathcal{A}^{\prime} .
$$

Thus, if $\delta=1$ then $q=0$, else the form (2.3) of $\left[\mathbf{E}_{1} \cdot \mathbf{n}\right](M)$ leads to the following boundary condition:

$$
\frac{\delta+1}{\delta-1} \frac{q(M)}{2 \epsilon_{0}}+\iint_{\partial \mathcal{A}^{\prime}} \frac{\mathbf{P M} \cdot \mathbf{n}(M) q(P) \mathrm{d} S_{P}^{\prime}}{4 \pi \epsilon_{0} P M^{3}}=\left(\operatorname{grad}\left[\phi_{0}\right] \cdot \mathbf{n}\right)(M), \quad M \in \partial \mathcal{A}^{\prime} .
$$


For a specified external potential $\phi_{0}$ the relation (2.5) actually provides a Fredholm boundary integral equation of the second kind for the unknown function $q$. Under the assumption $\delta>0$ the homogeneous form of (2.5) (take $\phi_{0}=0$ ) only admits the zero solution (since $\lambda=[1-\delta] /[1+\delta]$ lies in $]-1,1[$ it is not a characteristic value of the integral equation (B.25) discussed by Zabreyko (1975, p. 215)). Consequently, the application of the Fredholm theory to the weakly singular integral equation (2.5) (see Dautray \& Lions (1988)) implies that for $\phi_{0} \in H^{1}\left(\mathbb{R}^{3} \backslash \Omega\right)$ it admits one unique solution $q \in H^{-1 / 2}\left(\partial \mathcal{A}^{\prime}\right)$ if $H^{s}(\mathcal{U})$ denotes the usual Sobolev space. Thus, $(2.5)$ is a well-posed integral equation. Once its solution $q$ is obtained, one can further deduce the electrostatic potential and field both inside and around the body by invoking (2.1). Note that (2.4) together with the fact that the potential $\phi$ is harmonic in $\mathcal{A}^{\prime}$ ensure that the total polarization charge $Q$ is zero, that is,

$$
Q:=\iint_{\partial \mathcal{A}^{\prime}} q(P) \mathrm{d} S_{P}^{\prime}=0
$$

This explains why it is also worth giving the higher moments (dipole moments, for instance) of the unknown distribution $q$.

\section{Asymptotic expansion of the integral equation}

Under additional requirements regarding the body shape and the imposed electrostatic potential $\phi_{0}$ this section asymptotically expands (2.5) with respect to the small slenderness ratio. In order to clarify the added assumptions it is convenient to locate each point $M\left(x^{\prime}, y^{\prime}, z^{\prime}\right)$ by two new sets of dimensionless coordinates: a Cartesian set $(x, y, z)$ such that $x^{\prime}=e x, y^{\prime}=e y, z^{\prime}=L z$ and the attached cylindrical coordinates $(r, \theta, z)$ of axis $\left(z^{\prime \prime}, O^{\prime}, z^{\prime}\right)$ obeying $r^{2}=x^{2}+y^{2}$ and defining the usual and local vectors $\mathbf{e}_{r}$ and $\mathbf{e}_{\theta}$. Moreover, assume that $\partial \mathcal{A}^{\prime}$ is entirely described by a positive, single-valued and smooth enough shape function $f(\theta, z)=O(1)$ which is defined on $[0,2 \pi] \times[0,1]$ and such that each point $M\left(x^{\prime}, y^{\prime}, z^{\prime}\right)$ of $\partial \mathcal{A}^{\prime}$ can also be written $M(\theta, z)$ with $r=f(\theta, z)$. We note $\partial_{v}^{i} f=\partial^{i} f / \partial v^{i}$ for $v \in\{\theta, z\}$ and any positive integer $i$ and impose $\left|\partial_{\theta}^{1} f\right|=O(1),\left|f f_{z}^{1}\right|=O(1)$ on $\left.[0,2 \pi] \times\right] 0,1[$. Near the end points $z=0$ and $z=1$ the following behaviours are assumed:

$$
\begin{aligned}
& f^{2}(\theta, z)=\sum_{n \geqslant 1} c_{n}(\theta) z^{n} ; 2 f f_{z}^{1}(\theta, z)=\sum_{n \geqslant 1} n c_{n}(\theta) z^{n-1}, \quad z \rightarrow 0^{+}, \\
& f^{2}(\theta, z)=\sum_{n \geqslant 1} b_{n}(\theta)(1-z)^{n} ; 2 f f_{z}^{1}(\theta, z)=-\sum_{n \geqslant 1} n b_{n}(\theta)(1-z)^{n-1}, \quad z \rightarrow 1^{-},
\end{aligned}
$$

with smooth functions $c_{n}$ and $b_{n}$ such that $0<c_{1}(\theta)=O(1)$ and $0<b_{1}(\theta)=O(1)$. Note that (3.1), (3.2) indeed ensure that $f(\theta, 0)=f(\theta, 1)=0$. For $z \in] 0,1[$ the closed path $C(z)$ is the boundary of the non-dimensional cross-section $C s(z)=\{M(x, y, z)$ such that $\left.M(e x, e y, L z) \in \partial \mathcal{A}^{\prime}\right\}$ and $\mathbf{n}^{2 d}(M)$ designates the unit (two-dimensional) normal vector outward $C(z)$ at the point $M(\theta, z)$ of $C(z)$. With this notation one easily obtains, 
respectively for $M(\theta, z) \in \partial \mathcal{A}^{\prime}$ and $M(\theta, z) \in \partial \mathcal{A}^{\prime} \backslash\left\{O^{\prime}, E^{\prime}\right\}$,

$$
\mathbf{n}(M)=\frac{\mathbf{e}_{r}-f^{-1} f_{\theta}^{1} \mathbf{e}_{\theta}-\epsilon f_{z}^{1} \mathbf{e}_{z}}{s_{\epsilon}(\theta, z)} ; \quad \mathbf{n}^{2 d}(M)=\frac{\mathbf{e}_{r}-f^{-1} f_{\theta}^{1} \mathbf{e}_{\theta}}{s_{0}(\theta, z)}
$$

if the function $s_{t}$ obeys the following definition and property, for $P\left(\theta_{P}, z_{P}\right) \in \partial \mathcal{A}^{\prime}$ :

$$
s_{t}=\left\{1+\left(f^{-1} f_{\theta}^{1}\right)^{2}+\left(t f_{z}^{1}\right)^{2}\right\}^{1 / 2} ; \quad \mathrm{d} S_{P}^{\prime}=e L\left[f s_{\epsilon}\right]\left(\theta_{P}, z_{P}\right) \mathrm{d} \theta_{P} \mathrm{~d} z_{P} .
$$

The assumptions (3.1), (3.2) indeed give a sense to the above form of $\mathbf{n}(M)$ as the point $M$ approaches the end points $O^{\prime}$ and $E^{\prime}$ (one may check that $\mathbf{n}\left(E^{\prime}\right)=-\mathbf{n}\left(O^{\prime}\right)=\mathbf{e}_{z}$ ). Of course, if $M(\theta, z) \in\left\{O^{\prime}, E^{\prime}\right\}$ both the closed path $C(z)$ and the vector $\mathbf{n}^{2 d}(M)$ are not defined.

For each point $M(\theta, z)$ of $\partial \mathcal{A}^{\prime}$ recall that the integral $I[q](M)$ arising on the left-hand side of (2.5) is regular. This property authorizes us to express this quantity in terms of the small slenderness ratio $\epsilon$ and the $\operatorname{pair}(\theta, z)$ pertaining to $M$ by applying Fubini's theorem and employing for each point $P$ belonging to the body surface its new coordinates $\left(\theta_{P}, z_{P}\right)$. At a first stage the definitions (3.3) together with the link between $\left(\theta_{P}, x_{P}\right)$ and $\left(x_{P}, y_{P}\right)$ ensure that, for $M(\theta, z) \in \partial \mathcal{A}^{\prime} \backslash\left\{O^{\prime}, E^{\prime}\right\}$,

$$
\mathbf{P M} \cdot\left[f s_{\epsilon} \mathbf{n}\right](M)\left[q f s_{\epsilon}\right](P)=\epsilon_{0}\left\{\left[f s_{0}\right](M) \Delta(P, M)+\left(z_{P}-z\right)\left[f f_{z}^{1}\right](M)\right\} d(P),
$$

where the unknown density $d$ and the function $\Delta$ obey

$$
\begin{gathered}
\Delta(P, M)=\Delta\left(\theta_{P}, z_{P}, \theta, z\right)=\left\{\left[x-x\left(\theta_{P}, z_{P}\right)\right] \mathbf{e}_{x}+\left[y-y\left(\theta_{P}, z_{P}\right)\right] \mathbf{e}_{y}\right\} \cdot \mathbf{n}^{2 d}(M), \\
x\left(\theta_{P}, z_{P}\right)=f\left(\theta_{P}, z_{P}\right) \cos \theta_{P} ; y\left(\theta_{P}, z_{P}\right)=f\left(\theta_{P}, z_{P}\right) \sin \theta_{P} ; \epsilon_{0} d(P)=e\left[f s_{\epsilon} q\right](P) .
\end{gathered}
$$

Under the definitions

$$
h_{1}\left(\theta_{P}, z_{P}, \theta, z\right)=\Delta\left(\theta_{P}, z_{P}, \theta, z\right) ; h_{2}\left(\theta_{P}, z_{P}, \theta, z\right)=\left(z_{P}-z\right)\left[f f_{z}^{1}\right](\theta, z)
$$

it is straightforward to obtain, for $M(\theta, z) \in \partial \mathcal{A}^{\prime} \backslash\left\{O^{\prime}, E^{\prime}\right\}$,

$$
4 \pi e\left(f s_{\epsilon} I[q]\right)(M)=\epsilon^{2} \int_{0}^{2 \pi}\left\{A_{\theta_{P}, \epsilon}^{\theta, z}\left[h_{1}\left(\theta_{P}, z_{P}, \theta, z\right) d\right]+A_{\theta_{P}, \epsilon}^{\theta, z}\left[h_{2}\left(z_{P}, \theta, z\right) d\right]\right\} \mathrm{d} \theta_{P}
$$

if the new linear operator $A_{\theta_{P}, \epsilon}^{\theta, z}$ satisfies, for a smooth function $v$, the definition below:

$$
A_{\theta_{P}, \epsilon}^{\theta, z}[v]=\int_{0}^{1} \frac{v\left(\theta_{P}, z_{P}\right) \mathrm{d} z_{P}}{\left[\left(z_{P}-z\right)^{2}+\epsilon^{2} H^{2}\left(\theta_{P}, z_{P}, \theta, z\right)\right]^{3 / 2}}=\int_{-z}^{1-z} \frac{w(u) \mathrm{d} u}{\left[u^{2}+\epsilon^{2} h^{2}(u)\right]^{3 / 2}},
$$

where $w(u)=v\left(\theta_{P}, u+z\right), h(u)=H\left(\theta_{P}, u+z, \theta, z\right)$ and the function $H$ depends on the body shape function $f$ as follows:

$$
\begin{aligned}
H\left(\theta_{P}, z_{P}, \theta, z\right)= & \left\{\left[x(\theta, z)-x\left(\theta_{P}, z_{P}\right)\right]^{2}+\left[y(\theta, z)-y\left(\theta_{P}, z_{P}\right)\right]^{2}\right\}^{1 / 2} \\
& \times\left\{f^{2}\left(\theta_{P}, z_{P}\right)+f^{2}(\theta, z)-2 \cos \left(\theta_{P}-\theta\right) f\left(\theta_{P}, z_{P}\right) f(\theta, z)\right\}^{1 / 2} .
\end{aligned}
$$


Of course, it is possible to replace the domain of integration on the right-hand side of (3.9) by $[0,2 \pi] \backslash\{\theta\}$ and on this new set $H>0$. This explains why the integrals arising in definition (3.10) are regular for $\epsilon>0$. The form (3.9) clearly shows that the asymptotic estimate of $f s_{\epsilon} I[q]$ depends on the asymptotic behaviour of the quantity $A_{\theta P}^{\theta, z}[v]$. As outlined in Sellier (1997) it is not at all trivial to build such an asymptotic behaviour. Difficulties indeed occur because $A_{\theta_{P}, 0}^{\theta, z}[v]$ (here obtained by setting $\epsilon=0$ ) turns out to be hypersingular as soon as $w(0) \neq 0$. Moreover, the well-known procedures either available in the standard textbooks (see Bleistein \& Handelsman, 1975; Estrada \& Kanwal, 1994; Wong, 1989) or developed in the specific case of a constant function $h$ by other authors (see Handelsman \& Keller, 1967a; Moran, 1963; Geer, 1976) do not apply here any more. The method of matched asymptotic expansions (see Van Dyke (1975)) is likely to give the answer but the price to pay is a great deal of algebra and more and more tedious matching conditions to enforce as the order of approximation increases. In order to avoid such drawbacks the author has established in Sellier (1996) the asymptotic behaviour of a wider class of integrals depending upon a small or large parameter. The key tool for such a method is the concept of integration in the finite part sense of Hadamard (see Hadamard, 1932; Schwartz, 1966). Among the wide range of applications it is possible to obtain the required asymptotic behaviour of $A_{\theta,}^{\theta, z}[v]$ up to any order provided both functions $f$ and $h$ are smooth enough. For further details the reader is directed to 6 and to Sellier (1997). If $v_{m}=\operatorname{Max}_{[0,1]}|v|$, one thereafter gets the following asymptotic estimate:

$$
\int_{0}^{2 \pi} A_{\theta P, \epsilon}^{\theta, z}[v] \mathrm{d} \theta_{P}=I_{0}^{\theta, z}[v] / \epsilon^{2}+I_{1}^{z}[v] \log \epsilon+I_{2}^{\theta, z}[v]+O\left(v_{m} \epsilon^{2} \log \epsilon\right)
$$

where the linear operators $I_{0}^{\theta, z}, I_{1}^{z}$ and $I_{2}^{\theta, z}$ satisfy, for a smooth function $v\left(\theta_{P}, z_{P}\right)$,

$$
\begin{aligned}
I_{0}^{\theta, z}[v]= & \int_{0}^{2 \pi} \frac{2 v\left(\theta_{P}, z\right) \mathrm{d} \theta_{P}}{H^{2}\left(\theta_{P}, z, \theta, z\right)} ; I^{z}[v]=\int_{0}^{2 \pi} v\left(\theta_{P}, z\right) \mathrm{d} \theta_{P} ; I_{1}^{z}[v]=-\frac{\mathrm{d}^{2}}{\mathrm{~d} t^{2}}\left[I^{t}[v]\right]_{t=z} \\
I_{2}^{\theta, z}[v]= & f p \int_{0}^{1} \frac{I^{t}[v] \mathrm{d} t}{|t-z|^{3}}+(\log 2-1) \frac{\mathrm{d}^{2}}{\mathrm{~d} t^{2}}\left[I^{t}[v]\right]_{t=z} \\
& -\int_{0}^{2 \pi} \frac{\mathrm{d}^{2}}{\mathrm{~d} t^{2}}\left[\log \frac{H\left(\theta_{P}, z_{P}, \theta, z\right)}{2} v\left(\theta_{P}, z\right)\right]_{t=z} \mathrm{~d} \theta_{P} .
\end{aligned}
$$

In definition (3.14) the symbol $f p$ designates an integration in the finite part sense of Hadamard. Since it only involves the values of $v$ on the closed path $C(z)$ the term $I_{0}^{\theta, z}[v]$ is a two-dimensional quantity whilst the other operators $I_{1}^{z}$ and $I_{2}^{\theta, z}$ which require to know the function $v$ respectively on a neighbourhood of $C(z)$ and on the whole body surface are respectively weakly and strongly three-dimensional. Since $f s_{0}=O(1)$ and $\left|f f_{z}^{1}\right|=O(1)$ 
the integral equation (2.5) takes, for $M(\theta, z) \in \partial \mathcal{A}^{\prime} \backslash\left\{O^{\prime}, E^{\prime}\right\}$, the following form:

$$
\begin{aligned}
e\left[f s_{\epsilon} \mathbf{n} \cdot \operatorname{grad} \phi_{0}\right](M)= & {\left[f s_{0}\right](M)\left\{\frac{\delta+1}{\delta-1}\left[\frac{d}{2 f s_{0}}\right](M)+\frac{I_{0}^{\theta, z}[\Delta d]}{4 \pi}\right\} } \\
& +\frac{\epsilon^{2} \log \epsilon}{4 \pi}\left\{\left[f s_{0}\right](M) I_{1}^{z}[\Delta d]+\left[f f_{z}^{1}\right](M) I_{1}^{z}\left[\left(z_{P}-z\right) d\right]\right\} \\
& +\frac{\epsilon^{2}}{4 \pi}\left\{\left[f s_{0}\right](M) I_{2}^{\theta, z}[\Delta d]+\left[f f_{z}^{1}\right](M) I_{2}^{\theta, z}\left[\left(z_{P}-z\right) d\right]\right\} \\
& +O\left(d_{m} \epsilon^{4} \log \epsilon\right) .
\end{aligned}
$$

Accordingly, the next step consists in expanding the left-hand side, $\operatorname{LH}(M)$, of equation (3.15). This task requires that we detail the form of the electrostatic potential $\phi_{0}$ near the dielectric body. If $M(r, \theta, z)$ is near the boundary $\partial \mathcal{A}^{\prime}$ then $r=O(1)$ and application of the Fourier expansion theorem (see Geer (1976)) to the analytic function $\phi_{0}$ ensures the general representation

$$
\phi_{0}(M)=A_{0}\left(\epsilon^{2} r^{2}, z\right)+\sum_{n=1}^{\infty} \epsilon^{n} r^{n}\left\{A_{n}\left(\epsilon^{2} r^{2}, z\right) \cos n \theta+B_{n}\left(\epsilon^{2} r^{2}, z\right) \sin n \theta\right\}
$$

with functions $A_{n}(u, z)$ or $B_{n}(u, z)$ regular near $u=0$ for $0 \leqslant z \leqslant 1$. By superposition the study is thereafter restricted to the basic case

$$
\phi_{0}(M)=\phi_{0}(r, \theta, z)=\epsilon^{n} r^{n} \psi\left(\epsilon^{2} r^{2}, z\right) \cos n \theta, n \geqslant 0,
$$

where the smooth function $\psi(u, z)$ admits partial derivatives $\partial_{u}^{i} \partial_{z}^{j} \psi(u, z)=O(1)$ for $u$ near zero. Moreover, since $\phi_{0}$ is harmonic near the body those derivatives satisfy there the basic relation

$$
\partial_{z}^{2} \psi(u, z)+4(n+1) \partial_{u}^{1} \psi(u, z)+4 u \partial_{u}^{2} \psi(u, z)=0 .
$$

The forms (3.3) and (3.17) immediately yield, for $M(\theta, z) \in \partial \mathcal{A}^{\prime} \backslash\left\{O^{\prime}, E^{\prime}\right\}$ such that $r=f(\theta, z)$,

$$
\begin{aligned}
\mathrm{LH}(M):= & e\left[f s_{\epsilon} \mathbf{n} \cdot \operatorname{grad} \phi_{0}\right](M)=n \epsilon^{n} \psi\left(\epsilon^{2} r^{2}, z\right)\left[f^{n} \cos n \theta+f^{n-1} f_{\theta}^{1} \sin n \theta\right] \\
& -\epsilon^{n+2}\left[f^{n+1} f_{z}^{1} \partial_{z}^{1} \psi\left(\epsilon^{2} r^{2}, z\right)-2 f^{n+2} \partial_{u}^{1} \psi\left(\epsilon^{2} r^{2}, z\right)\right] \cos n \theta .
\end{aligned}
$$

Since $f=O(1)$ a Taylor expansion of (3.19) easily leads to

$$
\mathrm{LH}(M)=\epsilon^{n}\left\{a_{0}^{n}(M)+a_{1}^{n}(M) \epsilon^{2}+\left[a_{2}^{n}(M)+\delta_{n 0} O\left(\epsilon^{2}\right)\right] \epsilon^{4}\right\}
$$

with $\delta_{i j}$ denoting throughout the paper the usual Kronecker delta, $a_{i}^{n}(M)=O(1)$ for $i \in\{0,1,2\}$ and the useful relations

$$
\begin{aligned}
a_{0}^{n}(M)= & n \psi(0, z)\left[f^{n} \cos n \theta+f^{n-1} f_{\theta}^{1} \sin n \theta\right]=\psi(0, z) \partial_{\theta}^{1}\left[f^{n} \sin n \theta\right], \\
a_{1}^{n}(M)= & \partial_{u}^{1} \psi(0, z)\left[(n+2) f^{n+2} \cos n \theta+n f^{n+1} f_{\theta}^{1} \sin n \theta\right] \\
& -\partial_{z}^{1} \psi(0, z) f^{n+1} f_{z}^{1} \cos n \theta \\
a_{2}^{0}(M)= & 2 \partial_{u}^{2} \psi(0, z) f^{4}-\partial_{u}^{1} \partial_{z}^{1} \psi(0, z) f^{3} f_{z}^{1} .
\end{aligned}
$$


A Taylor expansion of (3.18) near $u=0$ also provides the following links:

$$
\partial_{z}^{2} \psi(0, z)+4(n+1) \partial_{u}^{1} \psi(0, z)=0 ; \quad \partial_{u}^{1} \partial_{z}^{2} \psi(0, z)+4(n+2) \partial_{u}^{2} \psi(0, z)=0 .
$$

Those relations make it possible to rewrite, for $n \geqslant 0$, the definitions (3.22), (3.23) as

$$
\begin{aligned}
& (n+2) a_{1}^{n}(M)=n \partial_{u}^{1} \psi(0, z) \partial_{\theta}^{1}\left[f^{n+2} \sin n \theta\right]-\partial_{z}^{1}\left[\partial_{z}^{1} \psi(0, z) f^{n+2}\right] \cos n \theta, \\
& 4 a_{2}^{0}(M)=-\partial_{z}^{1}\left[\partial_{u}^{1} \partial_{z}^{1} \psi(0, z) f^{4}\right] .
\end{aligned}
$$

Inspection of (3.15) suggests we introduce the unknown density $t(P)$ such that $\mathrm{d}(P)=\left[f s_{0} t\right](P)$. Observe that, on the non-dimensional closed path $C(z)$, the arc length $\mathrm{d} l_{P}$ can be written $\mathrm{d} l_{P}=\left[f s_{0}\right](P) \mathrm{d} \theta_{P}$. Finally, if the new operators $\mathcal{L}_{0, \delta}^{\theta, z}, \mathcal{L}_{1}^{z}$ and $\mathcal{L}_{2}^{\theta, z}$ satisfy, for $M(\theta, z) \in \partial \mathcal{A}^{\prime} \backslash\left\{O^{\prime}, E^{\prime}\right\}$,

$$
\begin{aligned}
& \mathcal{L}_{0, \delta}^{\theta, z}[u]=\frac{\delta+1}{\delta-1} \frac{u(\theta, z)}{2}+\frac{1}{2 \pi} \oint_{C(z)} \frac{\Delta\left(\theta_{P}, z, \theta, z\right) u(P) \mathrm{d} l_{P}}{H^{2}\left(\theta_{P}, z, \theta, z\right)}, \\
& \mathcal{L}_{1}^{z}[u]=I_{1}^{z}\left[f s_{0} u\right] /[4 \pi] ; \quad \mathcal{L}_{2}^{\theta, z}[u]=I_{2}^{\theta, z}\left[f s_{0} u\right] /[4 \pi],
\end{aligned}
$$

then the integral equation (2.5) admits the asymptotic form

$$
\begin{array}{r}
{\left[f s_{0}\right](M) \mathcal{L}_{0, \delta}^{\theta, z}[t]+\epsilon^{2} \log \epsilon\left\{\left[f s_{0}\right](M) \mathcal{L}_{1}^{z}[\Delta t]+\left[f f_{z}^{1}\right](M) \mathcal{L}_{1}^{z}\left[\left(z_{P}-z\right) t\right]\right\}} \\
+\epsilon^{2}\left\{\left[f s_{0}\right](M) \mathcal{L}_{2}^{\theta, z}[\Delta t]+\left[f f_{z}^{1}\right](M) \mathcal{L}_{2}^{\theta, z}\left[\left(z_{P}-z\right) t\right]\right\}+O\left(f s_{0} t_{m} \epsilon^{4} \log \epsilon\right) \\
=\epsilon^{n}\left\{a_{0}^{n}(M)+a_{1}^{n}(M) \epsilon^{2}+\left[a_{2}^{n}(M)+\delta_{n 0} O\left(\epsilon^{2}\right)\right] \epsilon^{4}\right\} .
\end{array}
$$

Observe that the property $f s_{0}=O(1)$ indeed makes it possible to write $d_{m}=O\left(t_{m}\right)$. Each other case

$$
\phi_{0}(M)=\phi_{0}(r, \theta, z)=\epsilon^{n} r^{n} \psi\left(\epsilon^{2} r^{2}, z\right) \sin n \theta, n \geqslant 1,
$$

may be handled (before setting the value of $n)$ by replacing the pair $(\cos n \theta, \sin n \theta)$ by $(\sin n \theta,-\cos n \theta)$ in (3.21)-(3.23), (3.25), (3.26) and subsequent results.

\section{Asymptotic estimates for the polarization surface-charge density and related quantities}

This section presents the asymptotic solution of the integral equation (2.5) if the external potential $\phi_{0}$ takes, near the slender body, the form (3.17) with $n \geqslant 0$. As explained below the asymptotic procedure will be restricted to the following cases: $\delta-1 \geqslant O(1) ; \delta=O(1)$ and $0<1-\delta=O(1) ; \delta-1=o(1)$.

\subsection{General remarks}

The form of the first term arising on the left-hand side of (3.29) suggests we consider, for $M(\theta, z) \in \partial \mathcal{A}^{\prime} \backslash\left\{O^{\prime}, E^{\prime}\right\}$, the integral equation

$$
\mathcal{L}_{0, \delta}^{\theta, z}[u]=a(M)=a(\theta, z),
$$


with a given function $a$ and an unknown solution $u$ defined on the dimensional closed path $C^{\prime}(z)=\left\{M\left(x^{\prime}, y^{\prime}, z^{\prime}\right) \in \partial \mathcal{A}^{\prime} \backslash\left\{O^{\prime}, E^{\prime}\right\}\right.$; with $\left.z^{\prime}=L z\right\}$. According to the definitions (3.6), (3.27) this equation (4.1) also reads

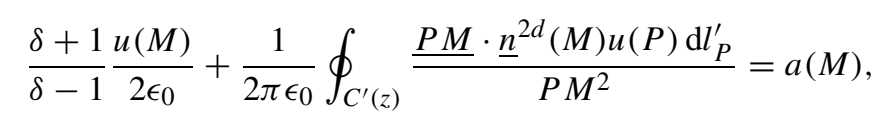

where $\mathrm{d} l_{P}^{\prime}=e \mathrm{~d} l_{P}$. This form (4.2) looks like the Fredholm integral equation of the second kind one might obtain when solving in the plane of the cross-section $C^{\prime}(z)$ the twodimensional counterpart of our 'dielectric' problem (2.1), (2.2) with $q^{2 d}=\epsilon_{0} u$ playing the role of the lineic-charge distribution on $C^{\prime}(z)$. However, there is here no reason for the 'total two-dimensional charge' $Q^{2 d}$, defined as

$$
Q^{2 d}=\epsilon_{0} e K^{z}[u] ; K^{z}[u]=\oint_{C(z)} u(P) \mathrm{d} l_{P},
$$

to vanish as would be the case for the polarization lineic-charge density attached to such a two-dimensional problem. More precisely, an integration of the equation (4.2) on the dimensional and closed path $C^{\prime}(z)$ immediately yields

$$
\oint_{C^{\prime}(z)} a(M) \mathrm{d} l_{M}^{\prime}=\frac{\delta+1}{\delta-1} \frac{e K^{z}[u]}{2}+\oint_{C^{\prime}(z)}\left\{\oint_{C^{\prime}(z)} \frac{P M}{\frac{P M}{n^{2 d}}(M) \mathrm{d} l_{M}^{\prime}} \underset{P M^{2}}{2 \pi} \frac{u(P)}{2 \pi} l_{P}^{\prime}\right.
$$

Since for any point $P$ of $C^{\prime}(z)$ the integral in braces equals $\pi$ it follows that any solution $u$ of (4.1) satisfies:

$$
\delta K^{z}[u]=\delta \oint_{C(z)} u(P) \mathrm{d} l_{P}=[\delta-1] \oint_{C(z)} a(P) \mathrm{d} l_{P} .
$$

Hence, for $\delta=0$ the integral equation (4.1) may admit a solution only if the compatibility condition $K^{z}[a]=0$ holds. Moreover, the associated homogeneous problem $\mathcal{L}_{0,0}^{\theta, z}[u]=0$ is known (use (4.2) or consult Zabreyko (1975, Chapter 7)) to have as solution any constant function $u$. Consequently, the integral equation

$$
\mathcal{L}_{0,0}^{\theta, z}[u]=a(M)
$$

is ill-posed: it does not possess a solution if $K^{z}[a] \neq 0$ and admits several solutions if $K^{z}[a]=0$. This feature prevents us from building, for small values of $\delta$, an asymptotic approximation to $t$ by employing the expansion (3.29). The link

$$
\mathcal{L}_{0, \delta}^{\theta, z}[u]=\mathcal{L}_{0,0}^{\theta, z}[u]+\frac{\delta u(M)}{\delta-1}
$$

indeed shows that, as $\delta$ goes to zero, the leading term on the left-hand side of (3.29) becomes $\left[f s_{0}\right](M) \mathcal{L}_{0,0}^{\theta, z}[t]$. Thus, the present method fails if $0 \leqslant \delta=o(1)$. For $\delta=0$, Sellier (1999) actually obtained the surface-charge density by working on a Fredholm integral equation of the first kind which is obtained by requiring the total electrostatic potential to have a constant but unknown value $V_{\epsilon}$ on the body surface. This constant 
$V_{\epsilon}$ and the function $q$ are therafter asymptotically expanded by combining the boundary integral equation and the global condition: $\iint_{\partial \mathcal{A}^{\prime}} q(P) \mathrm{d} S_{P}^{\prime}=0$. For $0<\delta=o(1)$ the problem is more tricky with this time an unknown potential function $\phi$ (see (2.1)) admitting near the body a general representation $\phi\left(A_{0}, A_{n}, B_{n} ; M\right)$ similar to (3.16) with functions $q, A_{0}, A_{n}$ and $B_{n}$ to be determined by enforcing the conditions (2.2) and $\phi(M)=\phi\left(A_{0}, A_{n}, B_{n} ; M\right)$ on $\partial \mathcal{A}^{\prime}$. Since such a task actually requires a whole study we disregard in the present paper the case $0<\delta=o(1)$. However, note that Barshinger \& Geer (1987) dealt with the cases $\delta=O(\epsilon)$ and $\delta=O\left(\epsilon^{2}\right)$ but for a dielectric body of revolution.

4.2 Case $1 . \delta-1 \geqslant O(1)$ or $\delta=O(1)$ and $0<1-\delta=O(1)$

For this case $\delta+1=O(\delta-1)$ and the density $d=f s_{0} t$ is written, for $t_{1 m}=\operatorname{Max}{ }_{[0,1]}\left|t_{1}\right|$,

$$
d=f s_{0} t=\epsilon^{n+2 \delta_{n}}\left\{f s_{0} t_{0}+\epsilon^{2} \log \epsilon\left[f s_{0} t_{1}\right]+\epsilon^{2}\left[f s_{0} t_{2}\right]+O\left(t_{1 m} \epsilon^{4} \log ^{2} \epsilon\right)\right\}
$$

with, in each cross-section, the following integral equations:

$$
\begin{aligned}
{\left[f s_{0}\right](M) \mathcal{L}_{0, \delta}^{\theta, z}\left[t_{0}\right]=} & a_{0+\delta_{n 0}}^{n}(M), \\
{\left[f s_{0}\right](M) \mathcal{L}_{0, \delta}^{\theta, z}\left[t_{1}\right]=} & -\left[f s_{0}\right](M) \mathcal{L}_{1}^{z}\left[\Delta t_{0}\right]-\left[f f_{z}^{1}\right](M) \mathcal{L}_{1}^{z}\left[(z P-z) t_{0}\right], \\
{\left[f s_{0}\right](M) \mathcal{L}_{0, \delta}^{\theta, z}\left[t_{2}\right]=} & a_{1+\delta_{n 0}}^{n}(M) \\
& -\left[f s_{0}\right](M) \mathcal{L}_{2}^{\theta, z}\left[\Delta t_{0}\right]-\left[f f_{z}^{1}\right](M) \mathcal{L}_{2}^{\theta, z}\left[\left(z_{P}-z\right) t_{0}\right]
\end{aligned}
$$

The pyramidal set of integral equations (4.9)-(4.11) is solved from the top to the bottom by inverting at each stage a kind of two-dimensional 'dielectric' problem. Note that the right-hand sides of (4.10) and (4.11) only depend on the first-order solution $t_{0}$ and require a careful application of the previous definitions of the function $\Delta$ and operators $\mathcal{L}_{1}^{z}$ and $\mathcal{L}_{2}^{\theta, z}$. For instance, the use of (4.5), (4.9), (3.13) together with (3.21) and (3.26) easily ensures that

$$
\begin{aligned}
& K^{z}\left[t_{0}\right]=\left[\frac{\delta-1}{\delta}\right] \int_{0}^{2 \pi} a_{n+\delta_{n 0}}^{n}(\theta, z) \mathrm{d} \theta=\delta_{n 0}\left[\frac{1-\delta}{2 \delta}\right] \frac{\mathrm{d}}{\mathrm{d} z}\left\{\int_{0}^{2 \pi} \partial_{z}^{1} \psi(0, z) f^{2}(\theta, z) \mathrm{d} \theta\right\}, \\
& \mathcal{L}_{1}^{z}\left[\left(z_{P}-z\right) t_{0}\right]=-\frac{1}{2 \pi} \frac{\mathrm{d}}{\mathrm{d} z}\left\{K^{z}\left[t_{0}\right]\right\}=\delta_{n 0}\left[\frac{\delta-1}{4 \pi \delta}\right] \frac{\mathrm{d}^{2}}{\mathrm{~d} z^{2}}\left\{\int_{0}^{2 \pi} \partial_{z}^{1} \psi(0, z) f^{2}(\theta, z) \mathrm{d} \theta\right\} .
\end{aligned}
$$

If $S(z)$ denotes the area of the non-dimensional cross-section $C s(z)$ the integral equations (4.10), (4.11) yield, after some algebra detailed in 6,

$$
\begin{aligned}
K^{z}\left[t_{1}\right]= & {\left[\frac{\delta-1}{2 \pi \delta}\right] \frac{\mathrm{d}}{\mathrm{d} z}\left\{S(z) \frac{\mathrm{d}}{\mathrm{d} z}\left(K^{z}\left[t_{0}\right]\right)\right\} ; \quad 2 S(z)=\int_{0}^{2 \pi} f^{2}(\theta, z) \mathrm{d} \theta, } \\
K^{z}\left[t_{2}\right]= & {\left[\frac{\delta-1}{2 \pi \delta}\right]\left\{-\frac{\mathrm{d}}{\mathrm{d} z}\left(S(z) \frac{\mathrm{d}}{\mathrm{d} z}\left(V_{z}\left[K^{t}\left[t_{0}\right]\right]\right)\right)+\int_{0}^{2 \pi}\left(2 \pi a_{1+\delta_{n 0}}^{n}\left(\theta_{P}, z\right)\right.\right.} \\
& \left.\left.+\frac{\mathrm{d}}{\mathrm{d} t}\left[\int_{C s(t)} \frac{\mathrm{d}}{\mathrm{d} t}\left\{\log H\left(\theta_{P}, t, \theta, z\right)\left[f s_{0} t_{0}\right]\left(\theta_{P}, t\right) \mathrm{d} S_{t}\right\}\right]_{t=z}\right) \mathrm{~d} \theta_{P}\right\},
\end{aligned}
$$


if the new operator $V_{z}$ obeys for any smooth enough function $\alpha(t)$

$$
V_{z}[\alpha(t)]=\alpha(z) \log 2+f p \int_{0}^{1} \frac{\alpha(t) \mathrm{d} t}{2|t-z|} .
$$

These equalities (4.12)-(4.15) hold for a body $\mathcal{A}^{\prime}$ whose shape is arbitrary.

4.3 Case 2. $\delta-1=\mu$ with $|\mu|=o(1)$

Owing to the following decomposition:

$$
\mathcal{L}_{0, \delta}^{\theta, z}[t]=\mathcal{L}_{0, \infty}^{\theta, z}[t]+t(M) / \mu
$$

the leading term occurring in (3.29) becomes $t(M) / \mu$ as $\mu \rightarrow 0$. In order to compare the different remaining terms one has to consider successively three different circumstances.

1. The case $|\delta-1|=|\mu| \gg \epsilon^{2}$. The reader may check that $d$ satisfies

$$
d(M)=\left[f s_{0} t\right](M)=\epsilon^{n+2 \delta_{n 0}}\left\{\mu a_{\delta_{n 0}}^{n}(M)-\mu^{2}\left[f s_{0}\right](M) \mathcal{L}_{0, \infty}^{\theta, z}\left[\frac{a_{\delta_{n 0}}^{n}}{f s_{0}}\right]+o\left(\mu^{2}\right)\right\}
$$

2. The case $|\delta-1|=O\left(\epsilon^{2}\right)$. This time one gets

$$
\begin{aligned}
d(M)= & \epsilon^{n+2 \delta_{n 0}}\left\{\mu a_{\delta_{n 0}}^{n}(M)-\mu^{2}\left[f s_{0}\right](M) \mathcal{L}_{0, \infty}^{\theta, z}\left[\frac{a_{\delta_{n 0}}^{n}}{f s_{0}}\right]\right. \\
& \left.+\mu \epsilon^{2} a_{1+\delta_{n 0}}^{n}(M)+o\left(\mu \epsilon^{2}\right)\right\} .
\end{aligned}
$$

3. The case $|\delta-1|=|\mu| \ll \epsilon^{2}$. Here, it is straightforward to obtain

$$
d(M)=\left[f s_{0} t\right](M)=\epsilon^{n+2 \delta_{n 0}}\left\{\mu a_{\delta_{n 0}}^{n}(M)+\mu \epsilon^{2} a_{1+\delta_{n 0}}^{n}(M)+o\left(\mu \epsilon^{2}\right)\right\} .
$$

Those results outline the necessity to handle the circumstances $\delta-1 \geqslant O(1)$ and $\delta-1=O(1)$ separately. Curiously, Barshinger \& Geer (1987) did not split the range $\delta \geqslant O(1)$ into these useful subdomains. It actually seems that the results they derived for $\delta \geqslant O(1)$ apply to the case $\delta-1 \geqslant O(1)$ only.

\subsection{Moments of the charge density}

Owing to the asymptotic estimate of the function $t$ it is also possible to expand the moments of the polarization charge density $q$. More precisely, for positive integers $i, j, k$ and $l$ we consider the moments $M_{l}(i, j, k)[q]$ of order $l$ and such that

$$
M_{l}(i, j, k)[q]=\iint_{\partial \mathcal{A}^{\prime}} x_{P}^{\prime i} y_{P}^{\prime j} z_{P}^{\prime k} q(P) \mathrm{d} S_{P}^{\prime}, \quad l=i+j+k \geqslant 0 .
$$


Our definitions of $f$ and $t$ immediately yield

$$
\frac{M_{l}(i, j, k)[q]}{\epsilon_{0} \mathrm{e}^{i+j} L^{k+1}}=\int_{0}^{1}\left\{\int_{0}^{2 \pi}[\cos \theta]^{i}[\sin \theta]^{j}\left[f^{i+j+1} s_{0} t\right](\theta, z) \mathrm{d} \theta\right\} z^{k} \mathrm{~d} z:=\mathcal{M}_{l}(i, j, k)[t]
$$

By employing the asymptotic form of $t$ (see (4.8) or (4.18)-(4.20)) the above relation easily provides the asymptotic behaviour of $M_{l}(i, j, k)[q]$. For instance, for case 1 , it immediately follows that

$$
\begin{aligned}
\frac{M_{l}(i, j, k)[q]}{\epsilon_{0} \mathrm{e}^{i+j} L^{k+1}}= & \epsilon^{n+2 \delta_{n 0}}\left\{\mathcal{M}_{l}(i, j, k)\left[t_{0}\right]+\mathcal{M}_{l}(i, j, k)\left[t_{1}\right] \epsilon^{2} \log \epsilon\right. \\
& \left.+\mathcal{M}_{l}(i, j, k)\left[t_{2}\right] \epsilon^{2}+O\left(\epsilon^{4} \log ^{2} \epsilon\right)\right\}
\end{aligned}
$$

Recall (see (2.6)) that the total charge $Q=\mathcal{M}_{0}(0,0,0)[q]=\epsilon_{0} L \int_{0}^{1} K^{z}[t] \mathrm{d} z$ is zero. Since

$$
K^{z}[t]=\int_{0}^{2 \pi} d(\theta, z) \mathrm{d} \theta ; K^{z}\left\{\mathcal{L}_{0, \infty}^{\theta, z}\left[\frac{a_{\delta_{n 0}}^{n}}{f s_{0}}\right]\right\}=\int_{0}^{2 \pi} a_{\delta_{n 0}}^{n}(\theta, z) \mathrm{d} \theta,
$$

and $f^{2}$ vanishes at the end points the equalities (3.21), (3.25), (3.26) together with (4.12), (4.14), (4.15) clearly show that the proposed asymptotic solutions (4.8) or (4.18)(4.20) agree with this global property. Among the moments of interest one often pays special attention to the dipole moment $\mathbf{D}[q]$ such that, if $\left(\mathbf{e}_{1}, \mathbf{e}_{2}, \mathbf{e}_{3}\right)=\left(\mathbf{e}_{x}, \mathbf{e}_{y}, \mathbf{e}_{z}\right)$,

$$
\mathbf{D}[q]=\iint_{\partial \mathcal{A}^{\prime}} q(P) \mathbf{O}^{\prime} \mathbf{P} \mathrm{d} S_{P}^{\prime}=\epsilon_{0} \sum_{m=1}^{3} \mathrm{e}^{1-\delta_{m 3}} L^{1+\delta_{m 3}} \mathcal{M}_{1}\left(\delta_{i m}, \delta_{j m}, \delta_{k m}\right)[t] \mathbf{e}_{m} .
$$

\section{Application to a slender dielectric body of elliptical cross-section}

At this stage it appears highly desirable to compare the proposed approach not only with the work of Barshinger \& Geer (1987) (that is, for a body of revolution) but also with more general cases. Since it is required to successively invert non-trivial Fredholm integral equations of the second kind this section only addresses case 1. Observe that a numerical treatment is likely to provide the solution of the pyramidal system (4.9)(4.11) without too much effort, that is, to yield the required density $d$ (see (4.8)) up to order $O\left(t_{1 m} \epsilon^{n+4+\delta_{n 0}} \log ^{2} \epsilon\right)$. However, it would also be nice to check the validity of each solution $t_{i}$ separately for $i \in\{0,1,2\}$. Unfortunately, such a refined comparison may only be achieved when closed forms exist for both the functions $t_{i}$ and the right density $d$. Among those special cases we consider a slender dielectric body $\mathcal{A}^{\prime}$ of elliptical crosssection embedded in a specific electrostatic potential $\phi_{0}$. More precisely, assume that $\partial \mathcal{A}^{\prime}$ is entirely defined by a smooth enough function $h$ that satisfies $h(0)=h(1)=0$ and a real number $0<\eta \leqslant 1$ such that, for $0<z<1$, each closed and non-dimensional path $\mathcal{C}(z)$ is the ellipse $\mathcal{E}(z)$ of the equation

$$
x^{2}+\frac{y^{2}}{\eta^{2}}=h^{2}(z)
$$


For comparison with the asymptotic behaviour of an analytical solution $d$ (see Appendix $\mathrm{C}$ ) we take for $\mathcal{A}^{\prime}$ an ellipsoid (that is, choose $h^{2}(z)=4 z(1-z)$ ) and impose the fourparameter electrostatic potential

$$
\phi_{0}(M)=\phi_{0}(x, y, z)=w_{0} z+\epsilon\left(w_{1} x+w_{1}^{\prime} x z\right)+2 w_{2} \epsilon^{2} x y .
$$

By using $\left(1 / \eta, \underline{e}_{y}, \underline{e}_{x}\right)$ instead of $\left(\eta, \underline{e}_{x}, \underline{e}_{y}\right)$ it is indeed possible to restrict attention to the case $0<\eta \leqslant 1$ and to this pecular form (5.2) for $\phi_{0}$ (terms $\epsilon w_{3} y$ or $\epsilon w_{3}^{\prime} y z$ are also tractable by interverting the variables $x$ and $y$ ). The decomposition (5.2) requires we handle at least three different cases:

$$
\begin{array}{ll}
\text { case (i): } & \phi_{0}(M)=\psi\left(\epsilon^{2} r^{2}, z\right)\left(\text { for }(5.2), \psi(u, z)=w_{0} z\right), \\
\text { case (ii): } & \phi_{0}(M)=\epsilon r \psi\left(\epsilon^{2} r^{2}, z\right) \cos \theta\left(\text { for }(5.2), \psi(u, z)=w_{1}+w_{1}^{\prime} z\right), \\
\text { case (iii): } & \phi_{0}(M)=\epsilon^{2} r^{2} \psi^{\prime}\left(\epsilon^{2} r^{2}, z\right) \sin 2 \theta\left(\text { for }(5.2), \psi^{\prime}(u, z)=w_{2}\right) .
\end{array}
$$

\subsection{Getting the solution $t_{0}$}

We first look at the general form of the integral equation

$$
\left[f s_{0}\right](M) \mathcal{L}_{0, \delta}^{\theta, z}[t]=a(\theta, z)
$$

here taken for the elliptical cross-section. If each point $P$ of $\mathcal{E}(z)$ is also located by its elliptical angle $\varphi_{P}$ such that

$$
x_{P}=h(z) \cos \varphi_{P}, \quad y_{P}=\eta h(z) \sin \varphi_{P}
$$

then the following useful relations hold, if both $M$ and $P$ belong to $\mathcal{E}(z)$ :

$$
\begin{aligned}
& P M^{2}=h^{2}(z)\left[1-\cos \left(\varphi_{P}-\varphi\right)\right]\left[1+\eta^{2}+\left(\eta^{2}-1\right) \cos \left(\varphi_{P}+\varphi\right)\right], \\
& h^{4}(z) \gamma^{2}(M)=x^{2}+y^{2} / \eta^{2} ; \quad \gamma(M) \underline{n}^{2 d}(M) \cdot \underline{P M}=1-\cos \left(\varphi_{P}-\varphi\right) .
\end{aligned}
$$

Note also that $f(\theta, z)=h(z) g_{\eta}(\theta)$ with

$$
g_{\eta}^{2}(\theta)=\frac{1+\tan ^{2} \theta}{1+\tan ^{2} \theta / \eta^{2}} ;\left[f s_{0}\right](M)=h^{2}(z) g_{\eta}^{2}(\theta) \gamma(M) ; \mathrm{d} l_{P}=\eta h^{2}(z) \gamma(P) \mathrm{d} \varphi_{P} .
$$

Substituting (5.8)-(5.10) into equation (5.6) immediately yields the equivalent integral equation

$$
\frac{\delta+1}{\delta-1} \frac{r(\varphi, z)}{2}+\frac{\eta}{2 \pi} \int_{0}^{2 \pi} \frac{r\left(\psi_{P}, z\right) \mathrm{d} \varphi_{P}}{1+\eta^{2}+\left(\eta^{2}-1\right) \cos \left(\varphi_{P}+\varphi\right)}=\frac{a(\theta, z)}{h^{2}(z) g_{\eta}^{2}(\theta)},
$$

provided the function $r$ is defined by:

$$
r(\psi, z)=\frac{t(\psi, z)}{h^{2}(z)}\left\{x^{2}+y^{2} / \eta^{4}\right\}^{1 / 2}=t(\psi, z) \gamma(M)
$$


Definition (5.12) introduces the functions $r_{i}$ associated to the unknown quantities $t_{i}$. By setting $\eta=1$ one recovers the case of a circular cross-section for which $\varphi=\theta$, $g_{\eta}=1, t=h(z) r$ and (5.11) admits a very simple form. When combined with the equalities (3.21), (3.22) the previous material leads (use, for instance, Gradshteyn \& Ryzhik (1965)) the following solutions $r_{0}$ :

$$
\begin{aligned}
r_{0}(M) & =\left[\frac{1-\delta}{2 \delta}\right] \frac{a(z)}{h^{2}(z)} \text { with } a(z):=\frac{\mathrm{d}}{\mathrm{d} z}\left[h^{2}(z) \partial_{z}^{1} \psi(0, z)\right] ; \text { case (i), } \\
r_{0}(M) & =\frac{2 \psi(0, z) \cos \varphi}{[\{(\delta+1) /(\delta-1)\}+\{(1-\eta) /(1+\eta)\}] h(z)} \\
& =\frac{2 \psi(0, z) x}{[\{(\delta+1) /(\delta-1)\}+\{(1-\eta) /(1+\eta)\}] h^{2}(z)} ; \text { case (ii) }, \\
r_{0}(M) & =\frac{2\left(1+\eta^{2}\right) \psi^{\prime}(0, z) \sin 2 \varphi}{\eta\left[\{(\delta+1) /(\delta-1)\}-\{(1-\eta) /(1+\eta)\}^{2}\right]} \\
& =\frac{4\left(1+\eta^{2}\right) \psi^{\prime}(0, z) x y}{\eta^{2}\left[\{(\delta+1) /(\delta-1)\}-\{(1-\eta) /(1+\eta)\}^{2}\right] h^{2}(z)} ; \text { case (iii) } .
\end{aligned}
$$

Note that under the conditions $\delta>0, \eta>0$ and $n \geqslant 1$ it is not possible to satisfy

$$
\frac{\delta+1}{\delta-1}=\left(\frac{1-\eta}{1+\eta}\right)^{n} \text { or } \frac{\delta+1}{\delta-1}=-\left(\frac{1-\eta}{1+\eta}\right)^{n} .
$$

This justifies the validity of relations (5.13)-(5.15) or incoming results (5.24)-(5.27) and (5.29), (5.30). For a circular cross-section (set $\eta=1$ ) the solution $t_{0}$ of (4.9) reads, for $n \geqslant 1$,

$$
t_{0}(M)=2 n\left[\frac{\delta-1}{\delta+1}\right] \psi(0, z) h^{n-1}(z) \cos n \theta \text { if } \psi_{0}(M)=\epsilon^{n} r^{n} \psi\left(\epsilon^{2} r^{2}, z\right) \cos n \theta .
$$

\subsection{The solution $t_{1}$}

This solution is gained without difficulty provided one notices that (successively combine (3.28), (3.6), (5.9) and (4.12))

$$
\begin{aligned}
& \mathcal{L}_{1}^{z}\left[\Delta t_{0}\right]=-\frac{\eta}{4 \pi \gamma(M)} \frac{\mathrm{d}^{2}}{\mathrm{~d} t^{2}}\left[\int_{0}^{2 \pi}\left[h(z)-h(t) \cos \left(\varphi_{P}-\varphi\right)\right] h^{2}(t) r_{0}\left(\varphi_{P}, t\right) \mathrm{d} \varphi_{P}\right]_{t=z}, \\
& \mathcal{L}_{1}^{z}\left[\left(z_{P}-z\right) t_{0}\right]=-\frac{1}{2 \pi} \frac{\mathrm{d} K^{z}\left[t_{0}\right]}{\mathrm{d} z}=\eta \delta_{n 0}\left[\frac{\delta-1}{2 \delta}\right] \frac{\mathrm{d}^{2}}{\mathrm{~d} z^{2}}\left\{h^{2}(z) \partial_{z}^{1} \psi(0, z)\right\} .
\end{aligned}
$$

More precisely, the results (5.13)-(5.15) yield $r_{1}(M)=0$ for case (iii) and also, if for $a(z)$ one keeps the definition (5.13),

$$
\begin{aligned}
& r_{1}(M)=-\frac{\eta}{4 h^{2}(z)}\left[\frac{\delta-1}{\delta}\right]^{2} \frac{\mathrm{d}}{\mathrm{d} z}\left\{h^{2}(z) \frac{\mathrm{d} a}{\mathrm{~d} z}\right\} ; \text { case (i), } \\
& r_{1}(M)=-\frac{\eta\left[h^{2}(z) \psi(0, z)\right]^{(2)}(z)}{[\{(\delta+1) /(\delta-1)\}+\{(1-\eta) /(1+\eta)\}]^{2}} \frac{\cos \varphi}{h(z)} ; \text { case (ii). }
\end{aligned}
$$


The case of the circular cross-section $(\eta=1)$ permits us once more to propose the solution $t_{1}=h r_{1}$ for $n \geqslant 1$. One actually gets for $\phi_{0}$ defined by (3.17) and $n \geqslant 1$ :

$$
t_{1}=-\delta_{n 1}\left[\frac{\delta-1}{\delta+1}\right]^{2} \frac{\mathrm{d}^{2}}{\mathrm{~d} z^{2}}\left\{h^{2}(z) \psi(0, z)\right\} \cos n \theta .
$$

\subsection{Form of the solution $t_{2}$}

Contrary to the previous solutions $t_{0}$ and $t_{1}$ the establishment of $t_{2}$ requires several steps of algebra. For convenience we set $t_{2}=t_{2}^{\prime}+t_{2}^{\prime \prime}$ with

$$
\left[f s_{0}\right](M) \mathcal{L}_{0, \delta}^{\theta, z}\left[t_{2}^{\prime}\right]=a_{1+\delta_{n 0}}^{n}(M)
$$

and the integral equations (B.4), (B.5) displayed in 6. Owing to the material available in this Appendix it is straightforward to check that $r_{2}^{\prime}=\gamma t_{2}^{\prime}$ reads

$$
\begin{aligned}
r_{2}^{\prime}(M)= & -\frac{\left[h^{4} \partial_{u}^{1} \partial_{z}^{1} \psi(0, z)\right]^{(1)}(z)}{4 h^{2}(z)}\left\{\left[\frac{1+\eta^{2}}{2}\right]\left[\frac{\delta-1}{\delta}\right]\right. \\
& \left.+\frac{\left(1-\eta^{2}\right) \cos 2 \varphi}{\{(\delta+1) /(\delta-1)\}+\{(1-\eta) /(1+\eta)\}^{2}}\right\} ; \text { case (i), } \\
r_{2}^{\prime}(M)= & \frac{2 \cos \varphi}{\{(\delta+1) /(\delta-1)\}+\{(1-\eta) /(1+\eta)\}}\left\{\left[\frac{1+3 \eta^{2}}{12}\right] h(z) \partial_{u}^{1} \psi(0, z)\right. \\
& \left.-\frac{1}{3 h^{2}(z)}\left[h^{3} \partial_{z}^{1} \psi(0, z)\right]^{(1)}(z)\right\}+\left[\frac{1-\eta^{2}}{2}\right] \\
& \times \frac{h(z) \partial_{u}^{1} \psi(0, z)}{\{(\delta+1) /(\delta-1)\}+\{(1-\eta) /(1+\eta)\}^{3}} \cos 3 \varphi ; \text { case (ii), } \\
r_{2}^{\prime}(M)= & \frac{2 \sin 2 \varphi}{\{(\delta+1) /(\delta-1)\}-\{(1-\eta) /(1+\eta)\}^{2}}\left\{\left[\frac{1+\eta^{4}}{2 \eta}\right] h^{2}(z) \partial_{u}^{1} \psi^{\prime}(0, z)\right. \\
& \left.-\frac{\eta}{4 h^{2}(z)}\left[h^{4}(z) \partial_{z}^{1} \psi^{\prime}(0, z)\right]^{(1)}(z)\right\}+\left[\frac{1-\eta^{4}}{2 \eta}\right] \\
& \times \frac{h^{2}(z) \partial_{u}^{1} \psi^{\prime}(0, z)}{\{(\delta+1) /(\delta-1)\}-\{(1-\eta) /(1+\eta)\}^{4}} \sin 4 \varphi, \text { case (iii). }
\end{aligned}
$$

The determination of $r_{2}^{\prime \prime}=\gamma t_{2}^{\prime \prime}$ requires further calculations whose main steps are detailed in 6. For case (i) the result takes the following form (the function $a$ keeps its definition (5.13)):

$$
\begin{aligned}
r_{2}^{\prime \prime}(M)= & \frac{\eta}{8 h^{2}(z)}\left[\frac{\delta-1}{\delta}\right]^{2}\left\{\frac{\mathrm{d}}{\mathrm{d} z}\left(h^{2}(z) O_{z}[a]\right)+\left(h^{2} a\right)^{(2)}(z)-\frac{\left[h^{2} a^{(2)}\right](z)}{2}\right. \\
& \left.+\left[h^{2} a^{(1)}\right]^{(1)}(z)\left(\log \left[\frac{\left(1+\eta^{2}\right) h^{2}(z)}{8 z(1-z)}\right]+2\right)-2\left[g a^{(1)}\right](z)-\left[a g^{(1)}\right](z)\right\} \\
& +4\left[\frac{1-\eta}{1+\eta}\right]\left[\frac{\delta}{\delta-1}\right] \frac{\left[h^{2} a^{(2)}\right](z) \cos 2 \varphi}{\{(\delta+1) /(\delta-1)\}+\{(1-\eta) /(1+\eta)\}^{2}} ; \text { case (i) }
\end{aligned}
$$


where the operator $O_{z}$ and the function $g$ obey

$$
\left[\frac{g}{h^{2}}\right](z)=\frac{1}{z}-\frac{1}{1-z} ; O_{z}[\alpha]=\left(\int_{0}^{1-z}-\int_{-z}^{0}\right)\left[\frac{\alpha(u+z)-\alpha(z)-\alpha^{(1)}(z) u}{u^{2}}\right] \mathrm{d} u .
$$

At this stage it is worth noting that the proposed asymptotic estimate (4.8) of $d$ holds throughout the domain $[0,1]$ if the quantities $d_{i}:=f s_{0} t_{i}=h^{2}(z) g_{\eta}^{2}(\theta) r_{i}$ remain of unit magnitude in this interval. Inspection of previous results (5.13)-(5.15), (5.20)-(5.22) and (5.24)-(5.26) clearly shows that this is true for $d_{0}, d_{1}$ and $d_{2}^{\prime}$ since $g_{\eta}(\theta)=O(1)$. The general assumptions bearing on $f$, that is, on $h$ (especially its behaviour (3.1), (3.2) near the end points) also ensure that $g=O(1)$ and $g^{(1)}=O(1)$ on $[0,1]$. This proves that $d_{2}^{\prime \prime}=O(1)$ for case (i). In case (ii) the calculations yield

$$
\begin{aligned}
r_{2}^{\prime \prime}(M)= & \frac{\eta h(z) \cos \varphi}{2 h^{2}(z)[\{(\delta+1) /(\delta-1)\}+\{(1-\eta) /(1+\eta)\}]^{2}}\left\{-\left(h^{2} b\right)^{(2)}(z)\right. \\
& \times\left(\log \left[\frac{\left(1+\eta^{2}\right) h^{2}}{8 z(1-z)}\right]+\frac{1}{2}\right)+O_{z}\left[\left(h^{2} b\right)^{(1)}\right]+\left[g b^{(1)}\right](z)+2\left[\frac{h^{(1)} b}{h}\right](z) \\
& \times\left[g-\left(h^{2}\right)^{(1)}\right](z)-2\left[h^{2} b^{(2)}\right](z)-10\left[h h^{(1)} b^{(1)}\right](z)-\frac{3}{2}\left[\left(h^{2}\right)^{(2)} b\right](z) \\
& \left.+\left[\frac{\eta-1}{\eta+1}\right]\left[2\left(h^{2} b\right)^{(2)}-\left(h^{2}\right)^{(2)} b\right](z)\right\}+\frac{\eta}{4}\left[\frac{\eta-1}{\eta+1}\right]^{2} \\
& \times\left\{\left[h b^{(2)}\right](z) \cos 3 \varphi\right\} /\{[\{(\delta+1) /(\delta-1)\}+\{(1-\eta) /(1+\eta)\}][\{(\delta+1) / \\
& \left.\left.(\delta-1)\}+\{(1-\eta) /(1+\eta)\}^{3}\right]\right\}, \quad b(z)=\psi(0, z) ; \text { case (ii). }
\end{aligned}
$$

As the reader may check the function $h^{(1)}\left[g-\left(h^{2}\right)^{(1)}\right]$ remains bounded throughout $[0,1]$ and this remark together with the result (5.29) ensure that $d_{2}^{\prime \prime}=h^{2}(z) g_{\eta}^{2}(\theta) r_{2}^{\prime \prime}=O(1)$ for case (ii) also. Finally, for case (iii) the solution $r_{2}^{\prime \prime}$ reads

$$
\begin{aligned}
r_{2}^{\prime \prime}(M)= & \left(\frac{1-\eta}{1+\eta}\right)^{3} \\
& \times\left\{\left[1+\eta^{2}\right]\left[h^{2} c^{(2)}\right](z) \sin 4 \varphi\right\} /\left\{6\left[\{(\delta+1) /(\delta-1)\}-\{(1-\eta) /(1+\eta)\}^{2}\right]\right. \\
& \left.\times\left[\{(\delta+1) /(\delta-1)\}-\{(1-\eta) /(1+\eta)\}^{4}\right]\right\} \\
& +\frac{\left[1+\eta^{2}\right] \sin 2 \varphi}{\left[\{(\delta+1) /(\delta-1)\}-\{(1-\eta) /(1+\eta)\}^{2}\right]^{2}} \\
& \times\left\{\frac{1+\eta^{2}}{(1+\eta)^{2}}\left[\left(h^{2}\right)^{(2)} c\right](z)+\frac{2\left(1+\eta^{2}-4 \eta\right)}{3\left(1+\eta^{2}\right)}\left[h^{2} c^{(2)}+6 h h^{(1)} c^{(1)}\right](z)\right\} .
\end{aligned}
$$

where $c(z)=\psi^{\prime}(0, z)$. These formulae (5.28)-(5.30) complete the determination of $r_{2}=r_{2}^{\prime}+r_{2}^{\prime \prime}$. Note that many simplifications occur for the circular cross-section $(\eta=1)$. Moreover, for $\eta=1$ and $\phi_{0}$ obeying the general form (3.17) with $n \geqslant 2$ one obtains the 
following solutions:

$$
\begin{aligned}
& t_{2}^{\prime}(M)=\frac{2}{n+2}\left[\frac{\delta-1}{\delta+1}\right]\left\{n^{2} h^{n+2}(z) \partial_{u}^{1} \psi(0, z)-\left[h^{n+2} \partial_{z}^{1} \psi(0, z)\right]^{(1)}(z)\right\} \frac{\cos n \theta}{h(z)}, \\
& t_{2}^{\prime \prime}(M)=\left[\frac{\delta-1}{\delta+1}\right]^{2}\left\{\frac{n^{2} h(z)}{n^{2}-1}\left[h^{n+2} \psi(0, z)\right]^{(2)}(z)-\left[h^{2}\left(h^{n} \psi(0, z)\right)^{(1)}\right]^{(1)}(z)\right\} \frac{\cos n \theta}{h(z)} .
\end{aligned}
$$

Hence, the asymptotic estimate (4.8) has been explicitly given in the case of a body of revolution if (3.17) holds with $n \geqslant 0$.

\subsection{Comparisons with an exact solution}

As 6 shows the exact density $d$ is available in a closed form when the applied potential $\phi_{0}$ satisfies (5.2) and for an ellipsoidal dielectric body such that (5.1) holds with $h^{2}(z)=$ $4 z(1-z)$. In these circumstances observe that $O_{z}\left[h^{2}\right]=O_{z}\left[\left(h^{2}\right)^{(1)}\right]=0$ and the previous results for $r_{0}, r_{1}, r_{2}^{\prime}$ and $r_{2}^{\prime \prime}$ ensure an asymptotic estimate of $d$ which perfectly agrees with the asymptotic behaviour of the exact solution (see (C.20)).

\section{Concluding remarks}

By taking $\delta \rightarrow \infty$ this work allows us to extend the results obtained in Sellier (1997) to the case of a general incoming flow (not necessarily uniform and of small incidence) around a slender body with rounded ends. Following Sellier (1997) it would also provide without too much additional effort the pressure coefficient on the body.

Observe that uniform asymptotic estimates are also easily obtained for the electrostatic potential and field both inside and outside the body by replacing in integral formulations (2.1) the polarization surface-charge density $q$ by its asymptotic approximation.

As explained the present theory addresses a 'straight' slender dielectric body of arbitrary cross-section. The more complicated case of a slender body of curved mean line would probably require a different and tedious treatment (think about the method of matched asymptotic expansions).

\section{REFERENCES}

BARShINGER, R. \& GEER, J. 1987 The electrostatic field about a slender dielectric body. SIAM J. Appl. Math. 47, 605-623.

Bleistein, N. \& Handelsman, R. A. 1975 Asymptotic Expansions of Integrals. New York: Holt, Rinehart \& Winston.

CADE, R. 1994 On integral equations of axisymmetric potential theory. IMA J. Appl. Math. 53, 1-25.

Dautray, R. \& Lions, J. L. 1988 Analyse Mathématique et Calcul Numérique. Paris: Masson.

Estrada, R. \& Kanwal, R. P. 1994 Asymptotic Analysis: A Distributional Approach. Boston: Birkhauser.

GEER, J. 1974 Uniform asymptotic solutions for the two-dimensional potential field about a slender body. SIAM J. Appl. Math. 26, 539-553.

GEER, J. 1975 Uniform asymptotic solutions for potential flow about a slender body of revolution. J. Fluid Mech. 67, 817-827. 
GeER, J. 1976 Stokes flow past a slender body of revolution. J. Fluid Mech. 78, 577-600.

Gradshteyn, I. S. \& RyzhiK, I. M. 1965 Tables of Integrals, Series and Products. London: Academic Press.

Hadamard, J. 1932 Lecture on Cauchy's Problem in Linear Differential Equations. New York: Dover.

Handelsman, A. \& Keller, J. B. 1967a Axially symmetric potential flow around a slender body. J. Fluid Mech. 28, 131-147.

Handelsman, A. \& Keller, J. B. 1967b The electrostatic field around a slender conducting body of revolution. SIAM J. Appl. Math. 15, 824-842.

JACKSON, J. D. 1975 Classical Electrodynamics. New York: Wiley.

Kellogg, O. D. 1953 Foundations of Potential Theory. New York: Dover.

LAMB, H. 1945 Hydrodynamics. New York: Dover.

MoRAN, J. 1963 Line source distributions and slender-body theory. J. Fluid Mech. 17, 285-303.

Schwartz, L. 1966 Théorie des Distributions. Paris: Hermann.

Sellier, A. 1996 Asymptotic expansion of a general integral. Proc. R. Soc. A 452, 2655-2690.

SELLIER, A. 1997 A general and formal slender-body theory in nonlifting case. Proc. R. Soc. A 453, $1733-1751$.

SELLIER, A. 1999 Asymptotic solution for the electrostatic field around a slender conducting body. IMA J. Appl. Math. 62, 167-193.

VAN Dy Ke, M. 1975 Perturbation Methods in Fluid Mechanics. Academic Press.

WoNG, R. 1989 Asymptotic Approximations of Integrals. Boston: Academic Press.

ZABREYKo, R. P. 1975 Integral Equations. Leyden: Noordhoff.

\section{Appendix A}

Consider $Q$ an integer, $N$ a positive integer such that $N \geqslant Q+1, w$ and $h$ two smooth enough functions and $K$ a ' $Q$ pseudo-homogeneous' kernel satisfying $K(\alpha u, \alpha v)=$ $\operatorname{sgn}(\alpha)|\alpha|^{Q} K(u, v)$. Under these assumptions the following asymptotic estimate holds (see Sellier (1996, Theorem 16)), for $z>0$ and $\epsilon \rightarrow 0^{+}$:

$$
\begin{aligned}
f p \int_{-z}^{1-z} w(u) K[u, \epsilon h(u)] \mathrm{d} u= & \sum_{n=0}^{N} \frac{\partial_{2}^{n} K(1,0)}{n !}\left[f p \int_{-z}^{1-z} \frac{\operatorname{sgn}(u) w(u) \mathrm{d} u}{u^{n-Q}[h(u)]^{-n}}\right] \epsilon^{n} \\
& +\sum_{m=0}^{N-Q-1} \sum_{l=0}^{m} \sum_{i=0}^{m-l} \frac{w^{(l)}(0) a_{m-l-i}^{i}}{l ! i !} \\
& \times\left[f p \int_{-\infty}^{\infty} \partial_{2}^{i} K[t, h(0)] t^{m} \mathrm{~d} t\right] \epsilon^{Q+m+1} \\
& -2 \sum_{n=0}^{N} \sum_{l=0}^{n} \sum_{i=0}^{n-l} \sum_{j=0}^{l-Q-1} \frac{w^{(j)}(0)[h(0)]^{l}}{l ! i ! j !} \\
& \times a_{l-Q-1-j}^{i} \partial_{2}^{n} K(1,0) \epsilon^{n} \log \epsilon+o\left(\epsilon^{N}\right)
\end{aligned}
$$

where $\partial_{2}^{n} K(u, v):=\partial^{n} K / \partial v^{n}, w^{(n)}(t):=\mathrm{d}^{n} w / \mathrm{d} t^{n}$ and the coefficients $a_{p}^{i}$ obey

$$
a_{p}^{0}=\delta_{p 0} ; \quad\{[h(u)-h(0)] / u\}^{i}=\sum_{p} a_{p}^{i} u^{p}, \text { as } u \rightarrow 0 \text { for } i \geqslant 1 .
$$


For further explanations regarding the symbol $f p$ which indicates an integration in the finite part sense of Hadamard the reader is directed to Sellier $(1996,1997)$. By choosing $K(u, v)=\left[u^{2}+v^{2}\right]^{-3 / 2}, Q=-3$ and $N=2$ one thereafter obtains

$$
\begin{aligned}
\int_{-z}^{1-z} & \frac{w(u) \mathrm{d} u}{\left[u^{2}+\epsilon^{2} h^{2}(u)\right]^{3 / 2}}=\frac{2 w(0)}{h^{2}(0)} \epsilon^{-2}-w^{(2)}(0) \log \epsilon+f p \int_{-z}^{1-z} \frac{\operatorname{sgn}(u) w(u) \mathrm{d} u}{u^{3}} \\
& +(1-\log 2) w^{(2)}(0)+[w(u) \log h(u)]^{(2)}(0)+O\left(w_{m} \epsilon^{2} \log \epsilon\right) .
\end{aligned}
$$

\section{Appendix B}

This Appendix is devoted to the establishment of (4.14), (4.15). It also details some of the steps used in getting the solution $t_{2}=t_{2}^{0}+t_{2}^{\prime \prime}$ (see Section $5 \mathrm{c}$ ).

Definitions (3.6) and (3.13) make it possible to cast (4.10) in the following form:

$$
4 \pi \mathcal{L}_{0, \delta}^{\theta, z}\left[t_{1}\right]=2\left[\frac{f f_{z}^{1}}{f s_{0}}\right](M) \frac{\mathrm{d} K^{z}\left[t_{0}\right]}{\mathrm{d} z}+\frac{\mathrm{d}^{2}}{\mathrm{~d} t^{2}}\left[\oint_{C(t)} \mathbf{n}^{2 d}(M) \cdot \mathbf{P M} t_{0}(P) \mathrm{d} l_{P}\right]_{t=z} .
$$

Consequently the link (4.5) yields

$$
K^{z}\left[t_{1}\right]=\frac{\delta-1}{2 \pi \delta}\left\{\frac{\mathrm{d} K^{z}\left[t_{0}\right]}{\mathrm{d} z} \frac{\mathrm{d} S}{\mathrm{~d} z}+\frac{\mathrm{d}^{2}}{\mathrm{~d} t^{2}}\left[\oint_{C(t)}\left(\oint_{C(z)} \frac{\mathbf{n}^{2 d}(M) \cdot \mathbf{P M}}{2} \mathrm{~d} l_{M}\right) t_{0}(P) \mathrm{d} l_{P}\right]_{t=z}\right\} .
$$

Finally our result (4.14) is easily deduced from (B.2) since

$$
2 S(z)=\oint_{C(z)} \Delta\left(\theta_{P}, t, \theta, z\right) \mathrm{d} l_{M}=\oint_{C(z)} \mathbf{n}^{2 d}(M) \cdot \mathbf{P M} \mathrm{d} l_{M} ; \quad \frac{\mathrm{d} S}{\mathrm{~d} z}=\int_{0}^{2 \pi} f f_{z}^{1}(M) \mathrm{d} \theta
$$

The derivation of (4.15) requires additional effort. Owing to (4.11), (3.14) and (3.28) we set $t_{2}=t_{2}^{0}+t_{2}^{1}+t_{2}^{2}$ with $\left[f s_{0}\right](M) \mathcal{L}_{0, \delta}^{\theta, z}\left[t_{2}^{0}\right]=a_{n+\delta_{n 0}}^{1}(M)$ and also

$$
\begin{aligned}
-4 \pi \mathcal{L}_{0, \delta}^{\theta, z}\left[t_{2}^{1}\right]= & {\left[\frac{f f_{z}^{1}}{f s_{0}}\right](M)\left\{(2 \log 2-2) \frac{\mathrm{d} K^{z}\left[t_{0}\right]}{\mathrm{d} z}+f p \int_{0}^{1} \frac{(t-z) K^{t}\left[t_{0}\right] \mathrm{d} t}{|t-z|^{3}}\right\} } \\
& +(\log 2-1) \int_{0}^{2 \pi} \frac{\mathrm{d}^{2}}{\mathrm{~d} t^{2}}\left[\Delta(P, M) f s_{0} t_{0}(P)\right]_{t=z} \mathrm{~d} \theta_{P} \\
& +f p \int_{0}^{1}\left[\oint_{C(t)} \frac{\Delta(P, M) t_{0}(P)}{|t-z|^{3}} \mathrm{~d} l_{P}\right] \mathrm{d} t . \\
4 \pi \mathcal{L}_{0, \delta}^{\theta, z}\left[t_{2}^{2}\right]= & \int_{0}^{2 \pi} \frac{\mathrm{d}^{2}}{\mathrm{~d} t^{2}}\left[\log H\left(\theta_{P}, t, \theta, z\right) \Delta(P, M)\left(f s_{0} t_{0}\right)(P)\right]_{t=z} \mathrm{~d} \theta_{P} \\
& +2\left[\frac{f f_{z}^{1}}{f s_{0}}\right](M) \int_{0}^{2 \pi} \frac{\mathrm{d}}{\mathrm{d} t}\left[\log H\left(\theta_{P}, t, \theta, z\right)\left(f s_{0} t_{0}\right)(P)\right]_{t=z} \mathrm{~d} \theta_{P} .
\end{aligned}
$$


The definition of the symbol $f p$ (see Hadamard (1932); Schwartz (1966)) yields, for any smooth enough function $\alpha(t)$, the basic relations

$$
\begin{gathered}
f p \int_{0}^{1} \frac{\operatorname{sgn}(t-z) \alpha(t) \mathrm{d} t}{(t-z)^{2}}=\frac{\mathrm{d}}{\mathrm{d} z}\left\{2 \alpha(z)+f p \int_{0}^{1} \frac{\alpha(t) \mathrm{d} t}{|t-z|}\right\} \\
=O_{z}[\alpha]+\alpha(z)\left[\frac{1}{z}-\frac{1}{1-z}\right]+\alpha^{(1)}(z) \log z(1-z) \\
2 f p \int_{0}^{1} \frac{\alpha(t) \mathrm{d} t}{|t-z|^{3}}=\frac{\mathrm{d}}{\mathrm{d} z}\left\{\frac{\mathrm{d} \alpha}{\mathrm{d} z}(z)+f p \int_{0}^{1} \frac{\operatorname{sgn}(t-z) \alpha(t) \mathrm{d} t}{(t-z)^{2}}\right\} \\
=O_{z}\left[\alpha^{(1)}\right]+\alpha^{(1)}(z)\left[\frac{1}{z}-\frac{1}{1-z}\right]+\alpha^{(2)}(z)[1+\log z(1-z)] \\
O_{z}[\alpha]=\left(\int_{0}^{1-z}-\int_{-z}^{0}\right)\left[\frac{\alpha(u+z)-\alpha(z)-\alpha^{(1)}(z) u}{u^{2}}\right] \mathrm{d} u
\end{gathered}
$$

The form (B.9) holds under the conditions $\alpha(0)=\alpha(1)=0$ and is deduced by integrating by parts. Hence, the combination of (4.5), (B.3), (B.4) and (B.6)-(B.8) immediately leads to (see the definition (4.16) of the operator $V_{z}$ )

$$
K^{z}\left[t_{2}^{1}\right]=\frac{1-\delta}{4 \pi \delta}\left\{S(z) \frac{\mathrm{d}^{2} K^{z}\left[t_{0}\right]}{\mathrm{d} z^{2}}+2 \frac{\mathrm{d}}{\mathrm{d} z}\left(S(z) \frac{\mathrm{d}}{\mathrm{d} z}\left(V_{z}\left[K^{t}\left[t_{0}\right]\right]\right)\right)\right\} .
$$

Observing that

$$
\oint_{C(z)} \log H\left(\theta_{P}, t, \theta, z\right) \mathbf{n}^{2 d}(M) \cdot \mathbf{P M} \mathrm{d} l_{M}=S(z)+2 \int_{C s(z)} \log H\left(\theta_{P}, t, \theta, z\right) \mathrm{d} S_{M},
$$

$\frac{\mathrm{d}}{\mathrm{d} t}\left[\int_{C S(t)} a(\theta, t) \mathrm{d} S_{t}\right]_{t=z}=\int_{0}^{2 \pi}\left[a f f_{z}^{1}\right](\theta, z) \mathrm{d} \theta+\int_{0}^{2 \pi}\left(\int_{0}^{f(\theta, z)}\left[\frac{\mathrm{d} a(\theta, t)}{\mathrm{d} t}\right]_{t=z} r \mathrm{~d} r\right) \mathrm{d} \theta$,

the relation (B.5) yields

$$
\begin{aligned}
K^{z}\left[t_{2}^{2}\right]= & \frac{\delta-1}{4 \pi \delta}\left\{S(z) \frac{\mathrm{d}^{2} K^{z}\left[t_{0}\right]}{\mathrm{d} z^{2}}\right. \\
& \left.+2 \int_{0}^{2 \pi} \frac{\mathrm{d}}{\mathrm{d} t}\left[\int_{C s(t)} \frac{\mathrm{d}}{\mathrm{d} t}\left(\log H\left(\theta_{P}, t, \theta, z\right)\left(f s_{0} t_{0}\right)\left(\theta_{P}, t\right)\right) \mathrm{d} S_{t}\right]_{t=z} \mathrm{~d} \theta_{P}\right\},
\end{aligned}
$$

and the equality (4.15) comes from the relations (B.8), (B.14) and the definition of $t_{2}^{0}$.

Now we look for $t_{2}^{j}$ for $j \in\{0,1,2\}$ and cases (i), (ii) and (iii) (see (5.3)-(5.5)). According to (5.21) and (5.11) one first introduces the quantities

$$
c_{n}(\theta, z):=a_{1+\delta_{n 0}}^{n}(M) /\left[h^{2}(z) g_{\eta}^{2}(\theta)\right], n \in\{0,1,2\},
$$


where $a_{2}^{0}, a_{1}^{1}$ respectively obey (3.26) and (3.25) but $a_{1}^{2}$ satisfies (3.25) with this time $(\cos 2 \theta, \sin 2 \theta)$ replaced by $(\sin 2 \theta,-\cos 2 \theta)$ (see the remark closing Section 3 ). Moreover, $f(\theta, z)=h(z) g_{\eta}(\theta)$ and $\eta \partial_{\theta}^{1}=g_{\eta}^{2}(\theta) \partial_{\varphi}^{1}$ (see (5.7)). It follows that:

$$
\begin{aligned}
\partial_{\theta}^{1}\left\{g_{\eta}^{3}(\theta) \sin \theta\right\} & =g_{\eta}^{2}(\theta) \partial_{\varphi}^{1}\left\{\sin \varphi+\left(\eta^{2}-1\right) \sin ^{3} \varphi\right\}, \\
\eta \partial_{\theta}^{1}\left\{g_{\eta}^{4}(\theta) \cos 2 \theta\right\} & =g_{\eta}^{2}(\theta) \partial_{\varphi}^{1}\left\{\cos ^{4} \varphi-\eta^{4} \sin ^{4} \varphi\right\} .
\end{aligned}
$$

Hence, one gets

$$
\begin{aligned}
4 h^{2}(z) c_{0}= & \left\{\frac{1+\eta^{2}}{2}+\frac{1+3 \eta^{2}}{4} h^{3}(z)\left[\frac{1-\eta^{2}}{2}\right] \cos 2 \varphi\right\}\left[h^{4}(z) \partial_{u}^{1} \partial_{z}^{1} \psi(0, z)\right]^{(1)}, \\
3 h^{2}(z) c_{1}= & \left\{\frac{1+3 \eta^{2}}{4} h^{3}(z) \partial_{u}^{1} \psi(0, z)-\left[h^{3} \partial_{z}^{1} \psi(0, z)\right\} \cos \varphi\right. \\
& +\frac{3}{4}\left(1-\eta^{2}\right) h^{3}(z) \partial_{u}^{1} \psi(0, z) \cos 3 \varphi, \\
4 h^{2}(z) c_{2}= & \left\{\frac{2\left(1+\eta^{4}\right)}{\eta} h^{4}(z) \partial_{u}^{1} \psi(0, z)-\eta\left[h^{4} \partial_{z}^{1} \psi^{\prime}(0, z)\right]^{(1)}(z)\right\} \sin 2 \varphi \\
& +\left[\frac{1-\eta^{4}}{\eta}\right] h^{4}(z) \partial_{u}^{1} \psi^{\prime}(0, z) \sin 4 \varphi,
\end{aligned}
$$

and this yields the proposed results (5.24)-(5.26). As regards $t_{2}^{1}$ we set (see the forms (5.13)-(5.15) taken by the function $r_{0}$ )

$$
r_{0}(\varphi, z)=\frac{v(z) b(\varphi)}{h(z)} ; I_{1}=\int_{0}^{2 \pi} b\left(\varphi_{P}\right) \mathrm{d} \varphi_{P} ; \quad I_{2}(\varphi)=\int_{0}^{2 \pi} \cos \left(\varphi_{P}-\varphi\right) b\left(\varphi_{P}\right) \mathrm{d} \varphi_{P} .
$$

With this notation observe that, for $M \in \mathcal{E}(z)$ and $P \in \mathcal{E}(t)$,

$$
\frac{K^{z}\left[t_{0}\right]}{\eta}=I_{1}[h v](z) ; \oint_{\mathcal{C}(t)} \Delta(M, P) t_{0}(P) \mathrm{d} l_{P}=\frac{\eta\left[h(z) I_{1}[h v](t)-I_{2}(\varphi)\left[h^{2} v\right](t)\right]}{h(z) \gamma(M)} .
$$

By combining (B.22), (B.6)-(B.7) and also (B.9) the integral equation (B.4) reads

$$
\begin{aligned}
\frac{4 \pi f s_{0} \mathcal{L}_{0, \delta}^{\theta, z}\left[t_{2}^{1}\right]}{\eta g_{\eta}^{2}(\theta)}= & -I_{1}\left\{\frac{\mathrm{d}}{\mathrm{d} z}\left(h^{2}(z) O_{z}[h v]+\left(h^{3} v\right)(z)\left[\frac{1}{z}-\frac{1}{1-z}\right]\right)+\left[h^{2}(h v)^{(2)}\right](z)\right. \\
& \left.+\left[h^{2}(h v)^{(1)}\right](z)\left[\frac{1}{z}-\frac{1}{1-z}\right]+\left[h^{2}(h v)^{(1)}\right]^{(1)}(z)(\log [4 z(1-z)]-2)\right\} \\
& +h(z) I_{2}(\varphi)\left\{O_{z}\left[h^{2} v\right]+\left(h^{2} v\right)^{(1)}(z)\left[\frac{1}{z}-\frac{1}{1-z}\right]\right. \\
& \left.+\left(h^{2} v\right)^{(2)}(z)\left(\log [4 z(1-z)]-\frac{1}{2}\right)\right\} .
\end{aligned}
$$

Note that $I_{2}(\varphi)=0$ for cases (i) and (iii) whereas $I_{2}(\varphi)=\pi \cos \varphi$ for case (ii). Accordingly, an easy inversion of the integral equation (B.23) immediately provides the 
function $t_{2}^{1}$. Moreover, for a smooth enough function $u(t, z)$ if we set $H=H\left(\theta_{P}, t, \theta, z\right)$ and $\mathcal{H}:=H\left(\theta_{P}, z, \theta, z\right)$ then

$$
\begin{aligned}
\partial_{t}^{2}[u \log H]_{t=z}= & \log \mathcal{H}\left[\partial_{t}^{2} u\right]_{t=z} \\
& +\left\{2\left[\partial_{t}^{1} u\right]_{t=z}+u(z, z)\left[\frac{h}{h^{(1)}}\left(\frac{h^{(1)}}{h}\right)^{(1)}\right](z)\right\} \partial_{t}^{1}[\log H]_{t=z} \\
& +\frac{u(z, z)}{H^{2}\left(\theta_{P}, z, \theta, z\right)}\left[\frac{h^{(1)}}{h}\right]^{2}(z) \\
& \times\left\{\mathbf{A P} \cdot \mathbf{A M}+2 \mathbf{P M} \cdot \mathbf{A P}-2\left(\frac{\mathbf{P M} \cdot \mathbf{A M}}{H\left(\theta_{P}, z, \theta, z\right)}\right)^{2}\right\},
\end{aligned}
$$

with points $M(f(\theta, z), z), P\left(f\left(\theta_{P}, z\right), z\right)$ and $A(0, z)$ belonging to the cross-section $\mathcal{C}(z)$. Thus, the integral equation (B.5) governing $t_{2}^{2}$ becomes

$$
\begin{aligned}
\frac{4 \pi f s_{0} \mathcal{L}_{0, \delta}^{\theta, z}\left[t_{2}^{2}\right]}{\eta g_{\eta}^{2}(\theta)}= & {\left[h^{2}(h v)^{(1)}\right]^{(1)}(z) M_{z}^{\varphi}[b]-\left[h\left(h^{2} v\right)^{(2)}\right](z) M_{z}^{\varphi}\left[b \cos \left(\varphi_{P}-\varphi\right)\right] } \\
& +\left[2 h\left(h^{2} v\right)^{(1)}+v \frac{h^{4}}{h^{(1)}}\left(\frac{h^{(1)}}{h}\right)^{(1)}\right](z) \frac{\mathrm{d}}{\mathrm{d} t}\left[\int_{0}^{2 \pi} \log H\left[1-\cos \left(\varphi_{P}-\varphi\right)\right]\right. \\
& \left.\times b\left(\varphi_{P}\right) \mathrm{d} \varphi_{P}\right]_{t=z}+2\left[h^{(1)}\right]^{2}(z)(h v)(z)\left\{\cos ^{2} \varphi\left(N_{z}^{\varphi}[b]-I_{\varphi}[b]\right)\right. \\
& +\eta^{2} \sin ^{2} \varphi\left(N_{z}^{\varphi}[b]-\eta^{2} J_{\varphi}[b]\right)-\eta^{2} \sin 2 \varphi K_{\varphi}[b] \\
& \left.-\frac{\cos \varphi}{2} N_{z}^{\varphi}\left[b \cos \varphi_{P}\right]-\frac{\eta^{2} \sin \varphi}{2} N_{z}^{\varphi}\left[b \sin \varphi_{P}\right]\right\}
\end{aligned}
$$

if the following definitions are adopted:

$$
\begin{aligned}
N_{z}^{\varphi}[a] & =\int_{0}^{2 \pi} \frac{a\left(\varphi_{P}\right) \mathrm{d} \varphi_{P}}{D\left(\varphi_{P}+\varphi\right)} ; \quad D(\alpha):=1+\eta^{2}+\left(\eta^{2}-1\right) \cos \alpha, \\
I_{\varphi}[a] & =\int_{0}^{2 \pi} \frac{\sin ^{2}\left[\left(\varphi_{P}+\varphi\right) / 2\right] \mathrm{d} \varphi_{P}}{D^{2}\left(\varphi_{P}+\varphi\right) /\left[2 a\left(\varphi_{P}\right)\right]} ; J_{\varphi}[a]=\int_{0}^{2 \pi} \frac{\cos ^{2}\left[\left(\varphi_{P}+\varphi\right) / 2\right] \mathrm{d} \varphi_{P}}{D^{2}\left(\varphi_{P}+\varphi\right) /\left[2 a\left(\varphi_{P}\right)\right]}, \\
K_{\varphi}[a] & =-\int_{0}^{2 \pi} \frac{\sin \left(\varphi_{P}+\varphi\right) \mathrm{d} \varphi_{P}}{D^{2}\left(\varphi_{P}+\varphi\right) / a\left(\varphi_{P}\right)} ; M_{z}^{\varphi}[a]=\int_{0}^{2 \pi} \log H\left(\theta_{P}, z, \theta, z\right) \mathrm{d} \varphi_{P} .
\end{aligned}
$$

By successively choosing $b(\alpha)=1, b(\alpha)=\cos \alpha$ and $b(\alpha)=\sin 2 \alpha$ those equalities (B.25)-(B.28) determine the function $t_{2}^{2}$ for each case (i), (ii) and (iii). Observe that $r_{2}^{\prime \prime}=\gamma(M)\left[t_{2}^{1}+t_{2}^{2}\right]$. For the sake of concision the calculations leading to the formulae (5.28), (5.29) are omittted.

\section{Appendix C}

This Appendix gives the exact polarization charge density $q$ (or $d$; see (3.7)) taking place on the boundary $\partial \mathcal{E}^{\prime}$ of an ellipsoidal body $\mathcal{E}^{\prime}$ for the specific applied potential $\phi_{0}$ defined 
by (5.2). This boundary $\partial \mathcal{E}^{\prime}$ admits, in terms of the non-dimensional coordinates $(x, y, z)$, the equation

$$
x^{2}+y^{2} / \eta^{2}=h^{2}(z)=4 z(1-z) .
$$

If one introduces the new notation

$$
X_{1}=e x ; \quad X_{2}=e y ; \quad X_{3}=L\left(z-\frac{1}{2}\right) ; \quad a_{1}=e ; \quad a_{2}=\eta e ; \quad 2 a_{3}=L ; \quad \epsilon=e / L
$$

the equation (C.1) and equality (5.2) successively become:

$$
\begin{aligned}
& \frac{X_{1}^{2}}{a_{1}^{2}}+\frac{X_{2}^{2}}{a_{2}^{2}}+\frac{X_{3}^{2}}{a_{3}^{2}}=1, \text { on } \partial \mathcal{E}^{\prime}, \\
& \phi_{0}(M)=\frac{\omega_{0}}{2}+\frac{1}{2 a_{3}}\left[\omega_{1}+\frac{\omega_{1}^{\prime}}{2}\right] X_{1}+\frac{\omega_{0} X_{3}}{2 a_{3}}+\frac{w_{1}^{\prime} X_{1} X_{3}}{4 a_{3}^{2}}+\frac{\omega_{2} X_{1} X_{2}}{2 a_{3}^{2}} .
\end{aligned}
$$

This latter form suggests we handle the special case $\phi_{0}(M)=\phi_{s}(M)=A X_{2}+B X_{1} X_{3}$. As proposed by Lamb (1945) we thus seek the total electrostatic potential $\phi$ (see (2.1)) both inside $\left(\phi_{1}\right)$ and outside $\left(\phi_{2}\right)$ the body $\mathcal{E}^{\prime}$ under the following forms:

$$
\begin{aligned}
& \phi_{1}(M)=X_{2}\left\{A+A^{\prime} \int_{0}^{\infty} \frac{S(v) \mathrm{d} v}{a_{2}^{2}+v}\right\}+X_{1} X_{3}\left\{B+B^{\prime} \int_{0}^{\infty} \frac{S(v) \mathrm{d} v}{\left(a_{1}^{2}+v\right)\left(a_{3}^{2}+v\right)}\right\}, \\
& \phi_{2}(M)=X_{2}\left\{A+A^{\prime} \int_{\lambda(M)}^{\infty} \frac{S(v) \mathrm{d} v}{a_{2}^{2}+v}\right\}+X_{1} X_{3}\left\{B+B^{\prime} \int_{\lambda(M)}^{\infty} \frac{S(v) \mathrm{d} v}{\left(a_{1}^{2}+v\right)\left(a_{3}^{2}+v\right)}\right\},
\end{aligned}
$$

if the functions $S$ and $\lambda(M)=\lambda\left(X_{1}, X_{2}, X_{3}\right)$ obey

$$
S(v)=\left\{\left(a_{1}^{2}+v\right)\left(a_{2}^{2}+v\right)\left(a_{3}^{2}+v\right)\right\}^{-1 / 2} ; \frac{X_{1}^{2}}{a_{1}^{2}+\lambda}+\frac{X_{2}^{2}}{a_{2}^{2}+\lambda}+\frac{X_{3}^{2}}{a_{3}^{2}+\lambda}=1,
$$

where it is understood that $\lambda$ is the greatest root of the equation (C.6) (see Lamb (1945)). Note that $\partial \mathcal{E}^{\prime}$ is described by $\lambda=0$ whilst $\lambda>0$ outside $\mathcal{E}^{\prime}$ and $\lambda<0$ inside $\mathcal{E}^{\prime}$. The choices (C.5), (C.6) ensure that $\phi$ is harmonic both inside and outside $\mathcal{E}^{\prime}$ (Lamb, 1945) and also continuous across the surface $\partial \mathcal{E}^{\prime}$. The unknown pair $\left(A^{\prime}, B^{\prime}\right)$ is finally determined by imposing the boundary condition (2.2). According to (C.3) and the definition (C.7) of $\lambda(M)$ it follows that

$$
\underline{n}(M)=\frac{\sum_{i=1}^{3} X_{i} / a_{i}^{2} \underline{e}_{i}}{\left[\left\{X_{1}^{2} / a_{1}^{4}\right\}+\left\{X_{2}^{2} / a_{2}^{4}\right\}+\left\{X_{3}^{2} / a_{3}^{4}\right\}\right]^{1 / 2}} ; \quad\left(\frac{\partial X_{i}}{\partial \lambda}\right)_{\lambda=0}=\frac{X_{i}}{2 a_{i}^{2}}
$$

and the condition (2.2) thereafter leads to

$$
A^{\prime}=\frac{a_{1} a_{2} a_{3} A}{\{2 \delta /(\delta-1)\}-\alpha_{2}} ; \quad B^{\prime}=\frac{a_{1} a_{2} a_{3}\left(a_{1}^{2}+a_{3}^{2}\right) B}{\{2 \delta /(\delta-1)\}-\beta_{2}}
$$


with the quantities $\delta, \alpha_{2}$ and $\beta_{2}$ such that

$$
\frac{\alpha_{2}}{a_{1} a_{2} a_{3}}=\int_{0}^{\infty} \frac{S(v) \mathrm{d} v}{a_{2}^{2}+v} ; \frac{\beta_{2}}{a_{1} a_{2} a_{3}\left(a_{1}^{2}+a_{3}^{2}\right)}=\int_{0}^{\infty} \frac{S(v) \mathrm{d} v}{\left(a_{1}^{2}+v\right)\left(a_{3}^{2}+v\right)} ; \quad \delta=\frac{\epsilon_{2}}{\epsilon_{1}} .
$$

After some additional calculations one obtains the associated density $q$ :

$$
\begin{aligned}
\frac{q}{\epsilon_{0}}(M)= & {\left[\operatorname{grad} \phi_{1}-\operatorname{grad} \phi_{2}\right] \cdot \mathbf{n}(M) } \\
= & \frac{2}{\left[\left\{X_{1}^{2} / a_{1}^{4}\right\}+\left\{X_{2}^{2} / a_{2}^{4}\right\}+\left\{X_{3}^{2} / a_{3}^{4}\right\}\right]^{1 / 2}}\left\{\frac{A X_{2}}{\left[\{2 \delta /(\delta-1)\}-\alpha_{2}\right] a_{2}^{2}}\right. \\
& \left.+\frac{B X_{1} X_{3}\left(a_{1}^{2}+a_{3}^{2}\right)}{\left[\{2 \delta /(\delta-1)\}-\beta_{2}\right] a_{1}^{2} a_{3}^{2}}\right\} .
\end{aligned}
$$

By combining the previous results (C.11) and the definitions (C.2) it is straightforward to deduce (via cyclical changes of indices) the polarization charge density $q$ pertaining to the imposed potential $\phi_{0}$ defined by (C.4). Observe that in the present circumstances

$$
d=f s_{0} t=\frac{e q}{\epsilon_{0}} f s_{\epsilon} ; f s_{\epsilon}=e g_{\eta}^{2}(\theta)\left\{\frac{X_{1}^{2}}{a_{1}^{4}}+\frac{X_{2}^{2}}{a_{2}^{4}}+\frac{X_{3}^{2}}{a_{3}^{4}}\right\}^{1 / 2} .
$$

Consequently the required density $d$ reads

$$
\begin{aligned}
\frac{d(M)}{g_{\eta}^{2}(\theta)}= & \epsilon x\left\{\frac{2 w_{1}+w_{1}^{\prime}}{\{2 \delta /(\delta-1)\}-I_{\epsilon}}+\frac{w_{1}^{\prime}(2 z-1)}{\{2 \delta /(\delta-1)\}\left\{1 /\left(1+4 \epsilon^{2}\right)\right\}-I_{\epsilon}^{\prime \prime}}\right\} \\
& +\epsilon^{2}\left\{\frac{4 w_{0}(2 z-1)}{\{2 \delta /(\delta-1)\}-I_{\epsilon}^{\prime}}+\frac{4 w_{2} x y}{\{2 \delta /(\delta-1)\}\left\{\eta^{2} /\left(1+\eta^{2}\right)\right\}-I_{\epsilon}^{\prime \prime \prime}}\right\},
\end{aligned}
$$

with the following definitions:

$$
\begin{array}{ll}
I_{\epsilon}=\int_{0}^{\infty} \frac{4 \eta \epsilon^{2}\left(t+4 \epsilon^{2}\right)^{-3 / 2} \mathrm{~d} t}{\left[(t+1)\left(t+4 \eta^{2} \epsilon^{2}\right)\right]^{1 / 2}} ; & I_{\epsilon}^{\prime}=\int_{0}^{\infty} \frac{4 \eta \epsilon^{2}(t+1)^{-3 / 2} \mathrm{~d} t}{\left[\left(t+4 \epsilon^{2} t\right)\left(t+4 \eta^{2} \epsilon^{2}\right)\right]^{1 / 2}}, \\
I_{\epsilon}^{\prime \prime}=\int_{0}^{\infty} \frac{4 \eta \epsilon^{2}\left(t+4 \eta^{2} \epsilon^{2}\right)^{-1 / 2} \mathrm{~d} t}{\left[(t+1)\left(t+4 \epsilon^{2}\right)\right]^{3 / 2}} ; & I_{\epsilon}^{\prime \prime \prime}=\int_{0}^{\infty} \frac{16 \eta^{3} \epsilon^{4}(t+1)^{-1 / 2} \mathrm{~d} t}{\left[\left(t+4 \epsilon^{2} t\right)\left(t+4 \eta^{2} \epsilon^{2}\right)\right]^{3 / 2}} .
\end{array}
$$

By using for instance Sellier (1996) it is possible to obtain the asymptotic expansion of the previous integrals as $\epsilon$ goes to zero. More precisely, the reader may check that

$$
\begin{aligned}
& I_{\epsilon}=\frac{2 \eta}{1+\eta}+4 \eta \epsilon^{2} \log \epsilon+4 \eta \epsilon^{2}\left\{\log \left[\frac{1+\eta}{2}\right]+\frac{3+\eta}{2(1+\eta)}\right\}+O\left(\epsilon^{4} \log \epsilon\right) \\
& I_{\epsilon}^{\prime}=8 \eta \epsilon^{2}\left\{-\log \epsilon+\log \left[\frac{2}{\eta+1}\right]-1\right\}+O\left(\epsilon^{4} \log \epsilon\right) \\
& I_{\epsilon}^{\prime \prime}=\frac{2 \eta}{1+\eta}+12 \eta \epsilon^{2} \log \epsilon+\eta \epsilon^{2}\left\{12 \log \left[\frac{1+\eta}{2}\right]+10+\frac{12}{(1+\eta)}\right\}+O\left(\epsilon^{4} \log \epsilon\right) \\
& I_{\epsilon}^{\prime \prime \prime}=\frac{2 \eta^{2}}{(1+\eta)^{2}}-\frac{4 \eta^{3} \epsilon^{2}}{(1+\eta)^{2}}+o\left(\epsilon^{2}\right) .
\end{aligned}
$$


Provided that $\delta-1 \geqslant O(1)$ or $\delta=O(1)$ and $0<1-\delta=O(1)$ the asymptotic estimate of $d$ thereafter reads

$$
\begin{aligned}
\frac{d(M)}{g_{\eta}^{2}(\theta)}= & 2 w_{0} \epsilon^{2}\left[\frac{\delta-1}{\delta}\right](2 z-1)\left\{1-4 \epsilon^{2}\left[\frac{\delta-1}{\delta}\right]\left[\log \epsilon+1+\log \left(\frac{1+\eta}{2}\right)\right]\right. \\
& \left.+O\left(\epsilon^{4} \log ^{2} \epsilon\right)\right\}+w_{2} \epsilon^{2}\left\{\frac{4 x y\left(1+\eta^{2}\right)}{\eta^{2}\left[\{(\delta+1) /(\delta-1)\}-\{(1-\eta) /(1+\eta)\}^{2}\right]}\right. \\
& \left.-\frac{16 x y\left(1+\eta^{2}\right)^{2}}{\eta\left(1+\eta^{2}\right)^{2}\left[\{(\delta+1) /(\delta-1)\}-\{(1-\eta) /(1+\eta)\}^{2}\right]^{2}}+o\left(\epsilon^{2}\right)\right\} \\
& +\frac{2 \epsilon x}{\{(\delta+1) /(\delta-1)\}+\{(1-\eta) /(1+\eta)\}}\left\{O\left(\epsilon^{4} \log ^{2} \epsilon\right)+w_{1}+w_{1}^{\prime} z\right. \\
& +2 w_{1}^{\prime} \epsilon^{2}(2 z-1)+\frac{4 \eta \epsilon^{2}}{\{(\delta+1) /(\delta-1)\}+\{(1-\eta) /(1+\eta)\}}\left[\left(w_{1}+\frac{w_{1}^{\prime}}{2}\right)\right. \\
& \times\left(\log \left[\frac{1+\eta}{2}\right]+\frac{3+\eta}{2(1+\eta)}\right)+\left(w_{1}+(3 z-1) w_{1}^{\prime}\right) \log \epsilon \\
& \left.\left.+\frac{w_{1}^{\prime}}{4}(2 z-1)\left[6 \log \left[\frac{1+\eta}{2}\right]+5\left(\frac{3+\eta}{1+\eta}\right)\right]\right]\right\} .
\end{aligned}
$$

